\title{
Hydrodynamic optimisation of small arrays of heaving point absorbers
}

\author{
Justin P. L. McGuinness ${ }^{1}{ }_{(D)}$. Gareth Thomas ${ }^{1}$
}

Received: 16 December 2015 / Accepted: 28 June 2016 / Published online: 13 July 2016

(C) Springer International Publishing Switzerland 2016

\begin{abstract}
This paper describes the optimisation of arrays of wave energy converters (WECs) of point absorber type. The WECs are spherical in shape and operate in heave only. Arrays of five to seven devices are considered. To simplify the optimisation, the arrays are constrained to lie in a specified geometry, namely a straight line or a circle, thereby reducing the number of array variables. The array layout is optimised from a hydrodynamic perspective with respect to the spacing or angles between the devices. Following the work of McGuinness and Thomas (Eleventh European Wave and Tidal Energy Conference. Nantes, France, 2015), the objective function of the optimisation is taken to be the mean of the interaction factor, rather than the interaction factor itself. This mean is defined over a non-dimensional length measure of the array. This is motivated by the desire to produce arrays that are stable to changes in the incident wavelength. A more general optimisation is performed here than in McGuinness and Thomas (Eleventh European Wave and Tidal Energy Conference. Nantes, France, 2015), with no prescribed symmetry in the arrays. The behaviour of the optimal arrays is analysed with respect to performance and device motions. Closely spaced groups of devices are found to exist in some of the optimal arrays; the implications of this and the possibility of replacing these groups with larger devices are discussed. For circular arrays, it is
\end{abstract}

Ph.D. funding for Justin P. L. McGuinness was provided by the Irish Research Council via a Government of Ireland Postgraduate Scholarship (GOIPG/2013/1197), and is gratefully acknowledged.

Justin P. L. McGuinness

j.mcguinness@umail.ucc.ie

Gareth Thomas

g.thomas@ucc.ie

1 Department of Applied Mathematics, University College Cork, Cork, Ireland shown that the inclusion of a further device at the circle centre tends to alter the overall performance of the array. Most optimal circular arrays formed a semi-circular pattern dependent on the incident wave direction. For all array geometries considered, it is seen that the incident wave angle has a large impact on the optimal layout and the overall performance.

Keywords Wave-power - Arrays · Optimisation · Interaction · Point absorber · Hydrodynamics

\section{Introduction}

The fundamental modelling of arrays of point absorber type wave power devices was presented independently by Evans (1979) and Falnes (1980). The point absorber approximation assumes that the ratio of device size to incident wavelength is small enough that the scattered wave field of the device may be neglected. This allows a simplification of calculations, particularly those relating to WEC arrays. Many subsequent papers have applied this theory to assess arrays of differing configurations, e.g. Thomas and Evans (1981), McIver (1994), Mavrakos and McIver (1997), Fitzgerald (2006), Fitzgerald and Thomas (2007) and Child (2011).

Linear arrays of five equally spaced devices were investigated by Thomas and Evans (1981) together with arrays of two such rows and arrays of thin-ship type WECs. Unequally spaced arrays of five devices were considered by McIver (1994) for both heave and surge motions and it was found that unequal spacing had a considerable effect on array performance. A question of interest was whether or not a linear array in head seas would behave as an attenuator, i.e. would the incident wave amplitude decay due to absorption as it passes down the array? The cited initial work demon- 
strates clearly that this is not the case and it was found, for configurations symmetric about the central point of the array, that array members worked with regard to a rule of symmetry. For example, for a five-member linear array, symmetric about the third member, the second and fourth members work equally hard and similarly for the first and fifth members.

General two-dimensional arrays of five devices were optimised by Fitzgerald (2006) and reported in Fitzgerald and Thomas (2007), where the array layouts were optimised by maximising the interaction factor in a point absorber regime. Arrays of heaving cylindrical WECs were similarly optimised using Genetic Algorithms by Child (2011), within a full interaction regime. Linear symmetric arrays of spherical point absorbers were optimised in McGuinness and Thomas (2015), where the mean of the interaction factor over the non-dimensional length of the array, rather than the interaction factor itself, was maximised.

As stated above, the concept of unequal spacing in a linear array was first considered by McIver (1994), who showed that unequally spaced arrays performed better in some cases in comparison to equally spaced arrays. However, McIver considered only a very specific case of unequal spacing, while subsequent research has moved to more general optimisations. The research presented herein is motivated by the possibility that unequally spaced linear arrays may perform better that their equally spaced analogs. The work of McGuinness and Thomas (2015) was similarly motivated, although considered only symmetric linear arrays of one spacing variable. An extension of this is presented here, where general non-symmetric linear arrays of five devices, described by three spacing variables, are optimised. This work is extended further to consider circular array geometries, with the WECs constrained to lie on the circumference and the possibility of including another device in the circle centre is also investigated. In these cases, the arrays are described by five angular variables, which the arrays are optimised with respect to.

One common issue encountered in Thomas and Evans (1981), Fitzgerald (2006) and Child (2011) is that the optimal array arrangements were often found to be only slightly different to those corresponding to very poorly performing arrays. Quite often, either the best and worst array layouts were surprisingly close or the optimal array had a sharp peak in performance surrounded by large troughs. This means that a small change in the non-dimensional parameters of such arrays, either by a physical mis-alignment or a change in sea conditions (incident wavelength or wave angle), can have a potentially disastrous impact on array performance. An important aspect of this paper is to optimise elementary array layouts, namely those constrained to a straight line or circle, such that the performance is stable to small changes in non-dimensional parameters. To this end, optimisation is performed by maximising the mean of the interaction factor over a non-dimensional length scale, rather than the interaction factor itself, with respect to the array layout.

Another item of concern when considering array performance is the motions of the individual devices associated with optimal performance. A hydrodynamically optimised array is typically accompanied by large amplitude device motions. The large motion of WECs creates engineering difficulties with the control, maintenance and power takeoff of the devices. In addition, linear wave theory assumes that all device motions are at most of the same order of the wave amplitude, and violation of this requirement invalidates the underlying assumptions; this is considered in Thomas and Evans (1981), Fitzgerald (2006), and McGuinness and Thomas (2015), where the optimal arrays were predicted to exhibit large device motions. Device motion constraints were investigated in Thomas and Evans (1981) and Fitzgerald (2006), and it was found that in some cases, these constraints severely limited array performance.

The present approach does not include full interactions and follows the point-absorber implementation employed in Evans (1979), Thomas and Evans (1981), McIver (1994), Fitzgerald (2006) and McGuinness and Thomas (2015). The accuracy of this approximation is discussed in Mavrakos and McIver (1997), where it is shown that the point absorber approximation gives almost exact agreement with the exact multiple scattering method for a non-dimensional device radius of $\mathrm{ka}<0.8$. Therefore, the work presented herein is conducted within this regime, namely for $\mathrm{ka}=0.4$. An external model is required in this methodology to determine the device motions and for the chosen device geometry, which is spherical in this case, the motions can be determined using the approach of Havelock (1955), for a fixed non-dimensional radius of the WECs.

\section{Mathematical formulation}

\subsection{Power absorption theory}

Consider an array of $N$ semi-submerged spheres, considered to be point absorbers and which operate in heave only. It is assumed that linear wave theory is applicable and that regular long-crested waves of amplitude $A$, frequency $\omega$, wavelength $\lambda$ and angle $\beta$ are incident on the array in water of infinite depth. It has been shown by Evans (1979) that the mean power absorbed by an array is given by

$P_{\text {abs }}=\frac{1}{8} \mathbf{X}^{\dagger} \mathbb{B}^{-1} \mathbf{X}-\frac{1}{2}\left(\mathbf{U}-\frac{1}{2} \mathbb{B}^{-1} \mathbf{X}\right)^{\dagger} \mathbb{B}\left(\mathbf{U}-\frac{1}{2} \mathbb{B}^{-1} \mathbf{X}\right)$, 
where $\mathbf{X}$ and $\mathbf{U}$ are complex time-independent column vectors of the exciting forces and velocities of the devices, respectively, $\mathbb{B}$ is the radiation damping matrix and ${ }^{\dagger}$ denotes complex conjugate transpose. In this notation, the exciting force and velocity of body $m$ are given by $\operatorname{Re}\left[X_{m} e^{-i \omega t}\right]$ and $\operatorname{Re}\left[U_{m} e^{-i \omega t}\right]$. The power absorbed can be maximised by taking the second term in (1) to be zero, which occurs when the velocity is

$\mathbf{U}_{\mathrm{opt}}=\frac{1}{2} \mathbb{B}^{-1} \mathbf{X}$

This gives the maximum power absorbed by the array to be

$P_{\text {opt }}=\frac{1}{8} \mathbf{X}^{\dagger} \mathbb{B}^{-1} \mathbf{X}$

The device displacement amplitudes are given by the nondimensional column vector $\mathbf{D}$, such that the displacement of the $m$ th device is given by $\operatorname{Re}\left[A D_{m} e^{-i \omega t}\right]$. Maximum power absorption occurs when

$\mathbf{D}_{\text {opt }}=\frac{i}{2 \omega A} \mathbb{B}^{-1} \mathbf{X}$.

An absolute measure of the power absorption is not ideal as this does not give an indication of the power absorption capabilities of a chosen array compared to other arrays or to an isolated device. Therefore, a relative power measure is first defined as the absorption length

$\mathcal{L}=\frac{P_{\text {abs }}}{P_{w}}$,

where $P_{w}$ is the mean power per unit crest width of incident wave. Equation (5) can be considered as the width of a wave train having the same mean energy as that extracted by the array. This can be optimised for heave motions, to give

$\mathcal{L}_{\text {opt }}=\frac{P_{\text {opt }}}{P_{w}}=\frac{\lambda}{2 \pi} N q$,

where $q$ is the interaction factor and can be written

$q=\frac{2 \pi}{\lambda N} \frac{P_{\mathrm{opt}}}{P_{w}}$.

The quantity $q$ is considered usually to be the key indicator of array performance and denotes the ratio of the performance of an array to that of $N$ isolated devices, thus quantifying the effect of deploying the devices in an array. Therefore, for heave motions alone, $q$ can also be expressed as

$q=\frac{P_{\mathrm{opt}}}{N P_{\mathrm{opt}}^{(1)}}$, where $P_{\mathrm{opt}}^{(1)}$ is the optimal power absorbed by a single isolated device. It can be shown that the interaction factor can be written in terms of the exciting forces alone, giving

$q=X_{m}^{\dagger}(\beta)\left[\frac{1}{2 \pi} \int_{0}^{2 \pi} X_{i}(\theta) X_{j}^{*}(\theta) \mathrm{d} \theta\right]_{m n}^{-1} X_{n}(\beta)$,

where ${ }^{*}$ denotes complex conjugate, repeated subscripts denote summation and $\left[a_{i j}\right]_{m n}^{-1}$ denotes the $(m, n)$ th term of the inverse of the matrix whose $(i, j) t h$ term is given in $\left[a_{i j}\right]$. A derivation of this result is given in Thomas and Evans (1981).

\subsection{Point absorber approximation}

In essence, the point absorber theory assumes that the devices are sufficiently small so that they do not produce a scattered wave field. The corresponding modelling assumption is that $k a \ll 1$, for a wavenumber $k$ and a device of radius $a$. A value of $k a=0.4$ has been assumed by previous authors and this is applied herein. Mavrakos and McIver (1997) showed that this value is well within the range of applicability of the point absorber approximation. This allows the far-field angular dependence of the WECs to be simplified, which in turn gives the following simplified expression for $q$, as shown in Evans (1979):

$q=\frac{1}{N} \ell^{\dagger} \mathbb{J}^{-1} \ell$

where $\ell$ is a column vector with components $\left\{\ell_{m}=\right.$ $\left.e^{i k d_{m} \cos \left(\beta-\alpha_{m}\right)} ; m=1, \ldots, N\right\}$, and $\mathbb{J}$ is an $N \times N$ matrix with elements $\mathbb{J}_{m n}=J_{0}\left(\mathrm{kd}_{m n}\right)$ for devices operating in heave, where $J_{0}(x)$ is the zeroth order Bessel function of first kind. In this notation, $d_{m}$ is the distance from the origin to the $m$ th devices, $d_{m n}$ is the distance between the $m$ th and $n$th devices, $\alpha_{m}$ is the angle from the origin to the $m$ th device measured positive in a counter-clockwise direction from the positive $x$ axis. In most (but not all) cases, it is convenient to fix one device at the origin of the system, usually the first device. In this case, $d_{m}$ can be considered as the distance between the first and $m$ th devices, and $\alpha_{m}$ the angle between the first and $m$ th devices.

An important consistency relation was derived for the $q$-factor by Fitzgerald (2006) and reported in Fitzgerald and Thomas (2007), where it was shown, within the point absorber regime for a single mode of motion, that

$\frac{1}{2 \pi} \int_{0}^{2 \pi} q(\beta) \mathrm{d} \beta=1$.

It can also be shown that a symmetry in the interaction factor with respect to the incident wave angle exists and is 
$q(\beta)=q(\beta+\pi)$.

For the case of linear arrays of physical length $L$, the above formulation can be simplified further. Since the angular dependence between each device is the same, a coordinate system can be used such that $\alpha_{m}=0$ for all devices. This gives the simpler expression $\ell_{m}=e^{i k d_{m} \cos \beta}$. Also, as consecutive device separations will be often used in the implementation, the convenient notation $s_{m}=d_{m(m+1)}$ is introduced.

For circular arrays of radius $r$, the origin is set to be the centre of the circle, thus $d_{m}=r$ for each device on the circle circumference and $d_{m}=0$ if the $m$ th device is in the centre of the circle.

\subsection{Optimisation method}

\subsubsection{Linear arrays}

Following McGuinness and Thomas (2015), the aim of the optimisation is to seek a layout of a linear array that is stable to changes in non-dimensional parameters associated with device spacing and incident wavelength. It should be noted that most previous studies assume an equal spacing $d$ between devices, determine $q$ for a given non-dimensional spacing parameter $\mathrm{kd}$ and then plot $q \mathrm{vs} \mathrm{kd}$ for a given range of $\mathrm{kd}$. In the present work, the objective function used in the optimisation is the mean of the interaction factor $q$ over a range of non-dimensional length $\mathrm{kL}$ and this is to be maximised. Thus the spacing is not specified but is to be determined. Denote the specified lower and upper bounds of the range of the non-dimensional array length to be $\mathrm{kL}_{l}$ and $\mathrm{kL}_{u}$, respectively. In terms of the above notation and explicitly including the variables upon which $q$ depends, the objective function can be formally written as

$$
\begin{aligned}
& I\left(\mathrm{ks}_{1}, \ldots, \mathrm{ks}_{N-1}\right) \\
& \quad=\frac{1}{\mathrm{~kL}_{u}-\mathrm{kL}_{l}} \int_{\mathrm{kL}_{l}}^{\mathrm{kL}_{u}} q\left(\mathrm{ks}_{1}, \ldots, \mathrm{ks}_{N-1}, \beta_{0}\right) \mathrm{d}[\mathrm{kL}],
\end{aligned}
$$

where $\beta_{0}$ is the prescribed fixed incident wave angle. It can be seen from (13) that the integration variable does not appear explicitly in the integrand but occurs via the individual parameters $\mathrm{ks}_{j}$.

It is expedient to introduce the following notation to simplify the calculations. The consecutive device separations are reparameterised as

$\mathrm{ks}_{j}=n_{j} \mathrm{~kL}$,

where $n_{j} \in(0,1)$ is a real parameter that represents the relative separation between devices with respect to the total length. Therefore, $\mathrm{ks}_{j}$ is written as a fraction of the total non-dimensional length giving

$$
\begin{aligned}
& I\left(n_{1}, \ldots, n_{N-1}\right) \\
& \quad=\frac{1}{\mathrm{~kL}_{u}-\mathrm{kL}_{l}} \int_{\mathrm{kL}_{l}}^{\mathrm{kL}_{u}} q\left(n_{1}, \ldots, n_{N-1}, \beta_{0}, \mathrm{~kL}\right) \mathrm{d}[\mathrm{kL}],
\end{aligned}
$$

and the variable of integration appears explicitly in the integrand. There are limits on the values of $n_{j}$; since the sum of all separations is the total length, $\sum_{j=1}^{N-1} \mathrm{ks}_{j}=\mathrm{kL}$ and consistency requires

$\sum_{j=1}^{N-1} n_{j}=1$

The optimisation algorithm is implemented in Fortran with the aid of Numerical Analysis Group (NAG) routines (http://www.nag.co.uk). The optimisation routine chosen to find the maximum of (15) is E04UCF, with appropriate algorithms employed for the calculation of Bessel functions, matrix inversion and quadrature. This algorithm searches for the minimum value of the objective function using a sequential quadratic programming method.

The optimisation is performed for several values of fixed wave angles $\beta_{0}$, namely $\beta_{0}=0, \frac{\pi}{4}, \frac{\pi}{2}$ in order to allow comparison with the work of McGuinness and Thomas (2015) and to assess the effect of different incident wave directions. Note that since the array is linear and lies on the $x$ axis, symmetry exists about $\beta=\frac{\pi}{2}$, as well as $\beta=\pi$. Hence, only angles between $\beta \in\left[0, \frac{\pi}{2}\right]$ need be investigated. Other wave angles in this range, such as $\beta_{0}=\frac{\pi}{8}, \frac{3 \pi}{8}$ were also investigated, but the results for these wave angles are not explicitly presented for brevity.

\subsubsection{Circular arrays}

Arrays are considered in which the WECs are constrained to lie in a circular geometry. For a circle of radius $r$ the array layout variables are the angular positions of each device on the circle. A convenient notation is introduced to define the relative angles $\theta_{j}$ between each device, so that

$\theta_{j}=\alpha_{j}-\alpha_{j+1}$

The possibility exists to place an extra device in the centre of the circle if desired. As one device will be at a fixed angular separation, to avoid replication of results, this can be used to remove one variable from the optimisation process. There are now $N-1$ variables, and consistency requires that 


$$
\sum_{j=1}^{N} \theta_{j}=2 \pi .
$$

In keeping with the preliminary unpublished work of C. Costigan (Modelling Circular Arrays of Wave-Power Devices, Project Report, University College Cork, 2014), the mean of the interaction factor is defined over a range of nondimensional radius $\mathrm{kr}$. By analogy with the previous section, denote the upper and lower bounds of the non-dimensional array radius as $\mathrm{kr}_{u}$ and $\mathrm{kr}_{l}$, respectively, and the objective function for circular arrays is written as

$$
\begin{aligned}
& I\left(\theta_{1}, \ldots, \theta_{N-1}\right) \\
& \quad=\frac{1}{\mathrm{kr}_{u}-\mathrm{kr}_{l}} \int_{\mathrm{kr}_{l}}^{\mathrm{kr}_{u}} q\left(\theta_{1}, \ldots, \theta_{N-1}, \beta_{0}, \mathrm{kr}\right) \mathrm{d}[\mathrm{kr}],
\end{aligned}
$$

for a fixed prescribed incident wave angle $\beta_{0}$. There are now $N-1$ optimisation variables which are the relative angles $\theta_{j}$ between the WECs. By averaging over kr in this manner, the aim is to find array layouts which perform well over a relatively large range of non-dimensional radius. This is done so that, for a fixed radius $r$, good performance is maintained even if the wavelength changes.

The optimisation is performed for $\beta_{0}=0, \frac{\pi}{4}, \frac{\pi}{2}$. Other angles, namely multiples of $\frac{\pi}{8}$, were also investigated but are not presented for brevity. As with the linear arrays in Sect. 2.3.1, NAG routines implemented in FORTRAN were used to evaluate the objective function and perform the optimisation.

\section{Optimisation of 5-device linear arrays}

In the most general case, this array is described by four variables, namely the position along the line of the second to fifth devices or the positions of three of these devices and the array length. However, in the optimisation procedure outlined above, the array length parameter $\mathrm{kL}$ is the variable of integration and the array is essentially described by three variables.

The arrays considered are general linear arrays without any stipulated symmetry and, therefore, the optimisation is performed with respect to three variables, namely $n_{1}, n_{2}, n_{3}$. The value of the remaining separation variable $\left(\mathrm{ks}_{4}\right.$ or $\left.n_{4}\right)$ is given by Eq. (16). In this way, the results of McGuinness and Thomas (2015) are extended to a more general formulation. It is assumed that the target non-dimensional length of a given array is $\mathrm{kL}=10$ and that the sea state under consideration is such that $\mathrm{kL}$ remains within the range $\left[\mathrm{kL}_{l}, \mathrm{~kL}_{u}\right]=[5,15]$. The method can be applied to any range of $\mathrm{kL}$ as required,

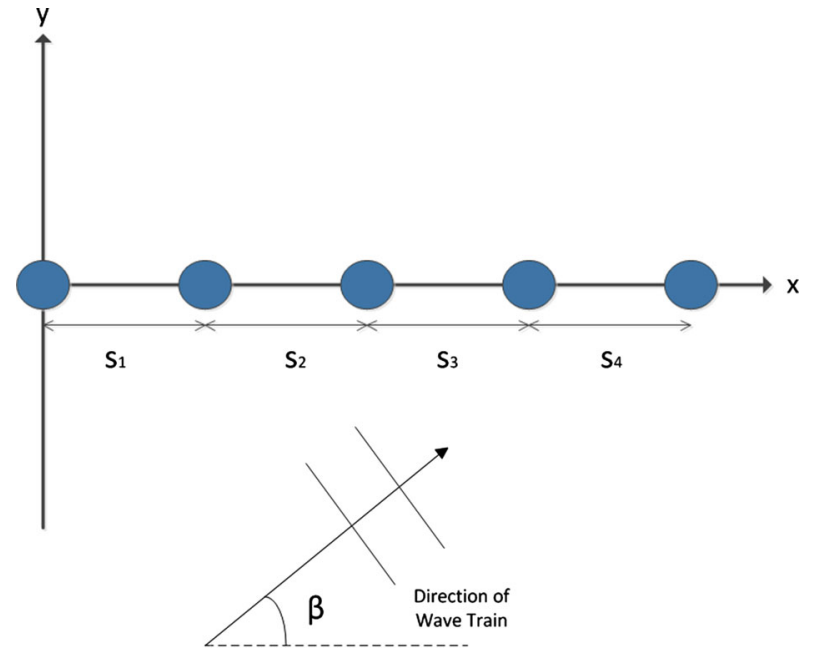

Fig. 1 Diagram of a general five-device linear array (without imposed symmetry)

as the values used in this work are chosen arbitrarily but are intended to represent a typical case.

A diagram of the array under consideration is shown in Fig. 1. Constraints on the device motions are not considered and the objective is simply to maximise the mean of the interaction factor with respect to the array layout, under the assumption of maximum power absorption.

A starting point is required as input to the optimisation algorithm. The interaction factor is acknowledged as being a highly oscillatory function, with many local maxima and minima. However, the results of this and previous work indicate that the mean of the interaction factor is a more well-behaved function for the case of linear arrays. Despite this, it is prudent to do an exhaustive search of the variable space for optimal values. This is conducted in a similar manner to Fitzgerald (2006), where the optimisation routine is run for a wide range of initial starting points for each variable. All permissible unique combinations of starting points with $n_{j}=\{0.1,0.2, \ldots, 0.7\}$ with $j=1, \ldots, 4$ were investigated, where some combinations were omitted due to consistency considerations in line with Eq. (16). For each value of $\beta_{0}$ investigated, the resulting optimal variable parameters and optimal mean interaction factor are then tabulated and presented. Diagrams of the layout of the best performing arrays in each case are also presented and analysed.

Minimum and maximum values of each separation parameter were enforced within the optimisation so that $0.05 \leq$ $n_{j} \leq 0.85$ for $j=1, \ldots, 4$. This ensures that no device will be within $5 \%$ of the total array length of another device. The upper bound of 0.85 was chosen to allow the possibility that all but one of the separations was exactly the minimum bound. This minimum bound is more limiting than that used in McGuinness and Thomas (2015), where a minimum value of 0.01 was allowed. It is unrealistic to allow devices to be 
positioned within $1 \%$ of the total array length of each other, as the devices will be touching/intersecting for all but very large array lengths. A $5 \%$ constraint was chosen here as it is a more feasible scenario. This value also avoided potential calculation difficulties due to numerical inaccuracies and poor behaviour of the objective function caused by small non-dimensional separation arguments.

The best optimal arrays found for each value of $\beta_{0}=$ $0, \frac{\pi}{4}, \frac{\pi}{2}$ are presented in Tables 2, 3 and 4 . Within these tables, arrays that were found to be similar to those presented, either by symmetry or by a negligible change in layout or performance, are omitted for brevity. For the best layout in each case, the behaviour of the optimal interaction factor is shown with respect to changes in $\mathrm{kL}$ and $\beta$ in Figs. 2 and 3, respectively. For comparison, the reader is referred to Thomas and Evans (1981) for results relating to a uniform linear array layout. Note that Thomas and Evans analyse the arrays with respect to the uniform spacing between the devices $\mathrm{kd}$, not the length of the array $\mathrm{kL}$; the relation between these is $\mathrm{kL}=4 \mathrm{kd}$. The average interaction factors for a uniform array are given in Table 1 for $\beta_{0}=0, \frac{\pi}{4}, \frac{\pi}{2}$.

\subsection{Head seas $\beta_{0}=0$}

Table 2 shows the optimal arrays determined by the algorithm, in descending order of performance. A diagram of the best array found for head seas is shown in Fig. 4, which is comprised of four devices grouped closely together at one side of the array, with a relatively isolated device at the other end. This array achieves an average interaction factor of $I=1.48$, which is considerably greater than unity; this is the best average value obtained for all the optimal linear arrays. From Fig. 2, it is clear that good performance is achieved

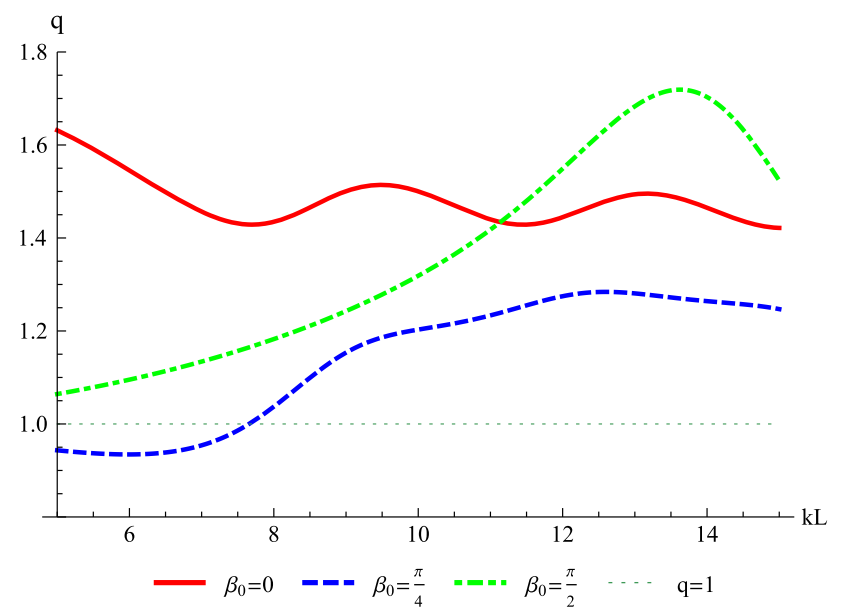

Fig. 2 Plots of optimal interaction factor $q$ vs non-dimensional length $\mathrm{kL}$ for the best linear arrays found for $\beta_{0}=0$ (solid curve), $\beta_{0}=\frac{\pi}{4}$ (dashed curve) and $\beta_{0}=\frac{\pi}{2}$ (dot-dashed curve). $q=1$ is shown by the thin dotted line

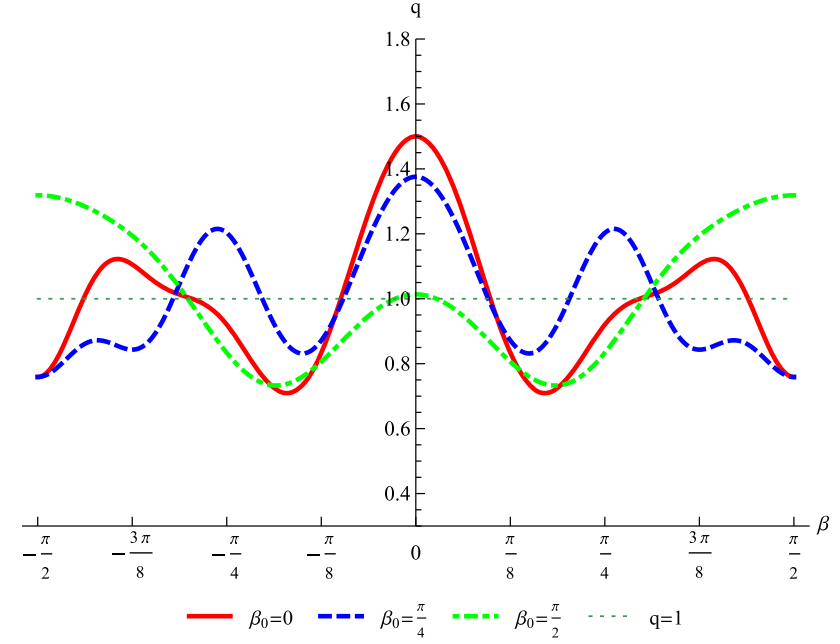

Fig. 3 Plots of optimal interaction factor $q$ vs incident wave angle $\beta$ for the best linear arrays found for $\beta_{0}=0$ (solid curve), $\beta_{0}=\frac{\pi}{4}$ (dashed curve) and $\beta_{0}=\frac{\pi}{2}$ (dot-dashed curve) with $\mathrm{kL}=10 . q=1$ is shown by the thin dotted line

Table 1 Average interaction factors $I$ for uniform linear array $\left(n_{1}=\right.$ $\left.n_{2}=n_{3}=n_{4}=0.25\right)$

\begin{tabular}{lll}
\hline & $\beta_{0}$ & $I$ \\
\hline Head seas & 0 & 1.0541 \\
Intermediate seas & $\frac{\pi}{4}$ & 0.9049 \\
Beam seas & $\frac{\pi}{2}$ & 1.3230 \\
\hline
\end{tabular}

Table 2 Optimal linear array parameters for $\beta_{0}=0$

\begin{tabular}{lllll}
\hline$n_{1}$ & $n_{2}$ & $n_{3}$ & $n_{4}$ & $I_{\mathrm{opt}}$ \\
\hline 0.05 & 0.05 & 0.05 & 0.85 & 1.4802 \\
0.05 & 0.85 & 0.05 & 0.05 & 1.3501 \\
0.05 & 0.45 & 0.45 & 0.05 & 1.2058 \\
\hline
\end{tabular}

Table 3 Optimal linear array parameters for $\beta_{0}=\frac{\pi}{4}$

\begin{tabular}{lllll}
\hline$n_{1}$ & $n_{2}$ & $n_{3}$ & $n_{4}$ & $I_{\mathrm{opt}}$ \\
\hline 0.05 & 0.85 & 0.05 & 0.05 & 1.1431 \\
0.05 & 0.45 & 0.45 & 0.05 & 1.1049 \\
0.05 & 0.05 & 0.05 & 0.85 & 0.8662 \\
\hline
\end{tabular}

over the entire domain considered, with $q \in[1.42,1.64]$ for $\mathrm{kL} \in[5,15]$, and the plot of $q$ can be seen to perform small oscillations about the average value of $q=1.48$. The range of variability of $q$ is also surprisingly small and this may be desirable so as to provide more certainty for a given WEC array developer.

Figure 3 shows that there is a range of approximately $\pm \frac{\pi}{8}$ around $\beta=0$ where $q$ remains greater than unity; outside this range, the array moves into areas of poor performance, 
Table 4 Optimal linear array parameters for $\beta_{0}=\frac{\pi}{2}$

\begin{tabular}{lllll}
\hline$n_{1}$ & $n_{2}$ & $n_{3}$ & $n_{4}$ & $I_{\mathrm{opt}}$ \\
\hline 0.0500 & 0.2252 & 0.3859 & 0.3359 & 1.3643 \\
0.3419 & 0.1581 & 0.1581 & 0.3419 & 1.3437 \\
\hline
\end{tabular}

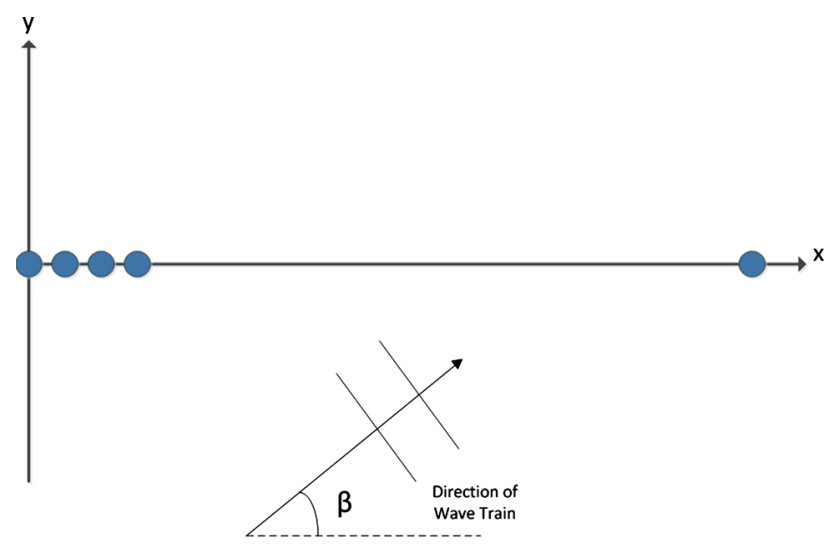

Fig. 4 Diagram of most optimal linear array found for $\beta_{0}=0$

as poor as $q \approx 0.7$. This behaviour is quite similar to that observed by McGuinness and Thomas (2015), for some of the arrays optimised in head seas. In this case, however, a greater peak in $q$ is obtained, which is also accompanied by larger troughs at non-optimal wave angles, most likely due to the increased freedom of the array layout within the optimisation routine.

Figure 5 shows the optimal displacements of the WECs, given by Eq. (4), within the optimal array for $\beta_{0}=0$. As expected, the optimal displacements are unacceptably large $(D>100)$ for the grouped devices, agreeing with the results of McGuinness and Thomas (2015) and earlier authors. This is enhanced for the smaller non-dimensional array lengths, where the predicted optimal displacements increase with decreasing $\mathrm{kL}$. The isolated device exhibits more reasonable motions of $D \approx 3$ for the entire domain.

It should be noted that the array layout obtained here is highly dependent on the constraints and consistency relations imposed within the calculations. First, all of the optimal separation parameters are at their maximum or minimum allowable values. This suggests that if these variable constraints were altered, the resulting optimal array layout and performance would also change. Calculations have been performed with different minimum values of $n_{j}$ and in each case the optimal array tended to the same optimal layout, or an equivalent one due to symmetry, with $n_{1}, n_{2}$ and $n_{3}$ at the minimum allowed values. Second, the isolated device in these optimal array layouts is an artefact of the formulation employed, particularly via (16). In the optimal case, this consistency forces one of the separations to be considerably larger than the others to preserve the total length of the array. It may be the case that allowing the fifth device to be closer

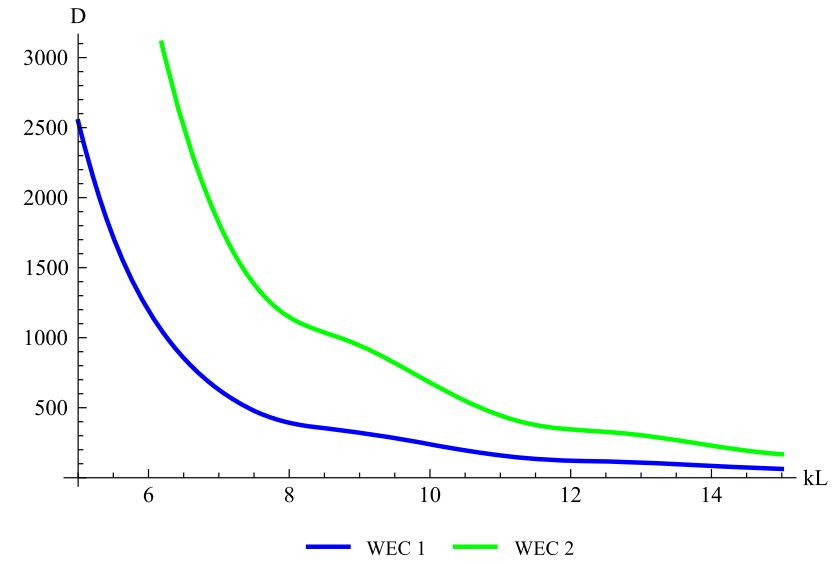

(a) Grouped WECs

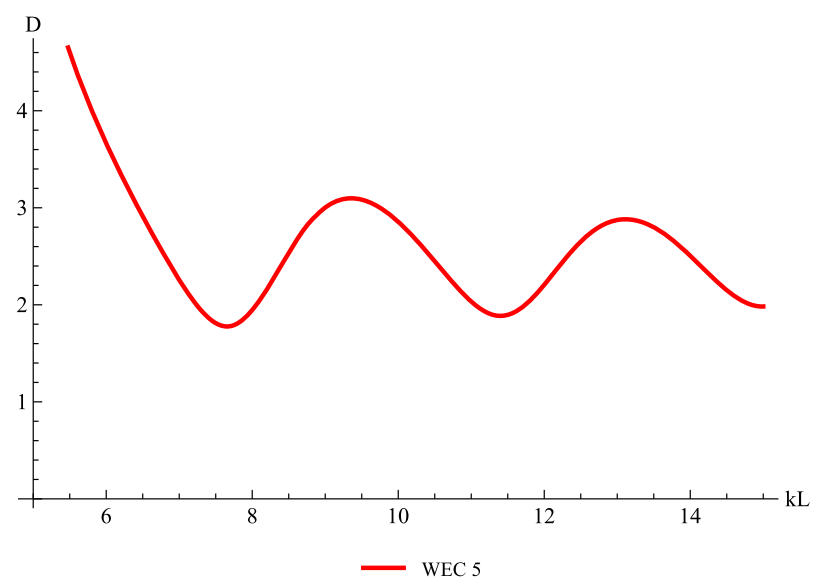

(b) Isolated WEC

Fig. 5 Optimal displacements for best optimised linear array with $\beta_{0}=0$. The displacements of WECs 3 and 4 are very similar to WECs 2 and 1 respectively and are thus omitted from this figure for clarity

to the group may give improved performance, although it is acknowledged that this would cause deployment difficulties.

In the case of head seas, the incident wave direction is parallel to the line of devices and the optimisation pushes all the devices as close together as possible, with one device preserving the total length of the array. This is likely due to the fact that the radiating wave amplitude decreases as it moves away from the originating WEC. Therefore, since optimal unconstrained device motions are assumed, the optimisation wants to place the WECs as close as possible to maximise the constructive interference due to the radiated wave field. This physical interpretation applies to linear arrays in head seas since the wave direction and the interaction direction are the same.

\subsection{The intermediate angle $\beta_{0}=\frac{\pi}{4}$}

The optimisation results for $\beta_{0}=\frac{\pi}{4}$ are shown in Table 3 . The best array in this case involves two separated groups of 


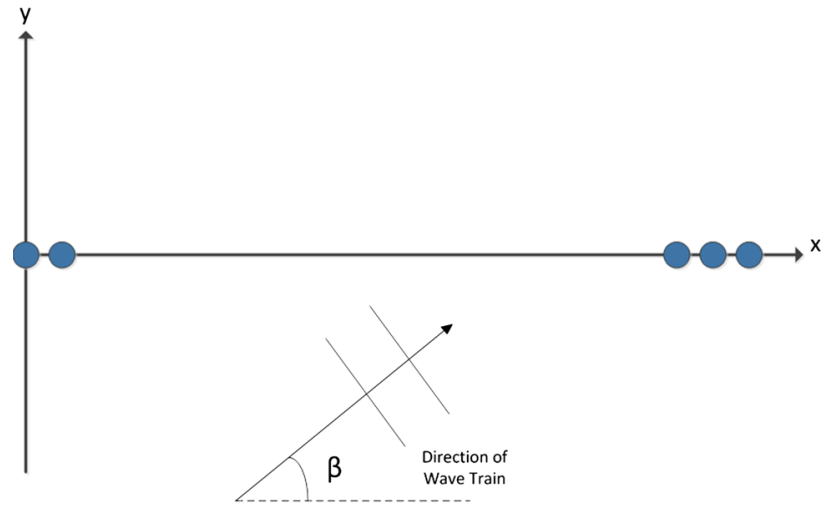

Fig. 6 Diagram of most optimal linear array found for $\beta_{0}=\frac{\pi}{4}$

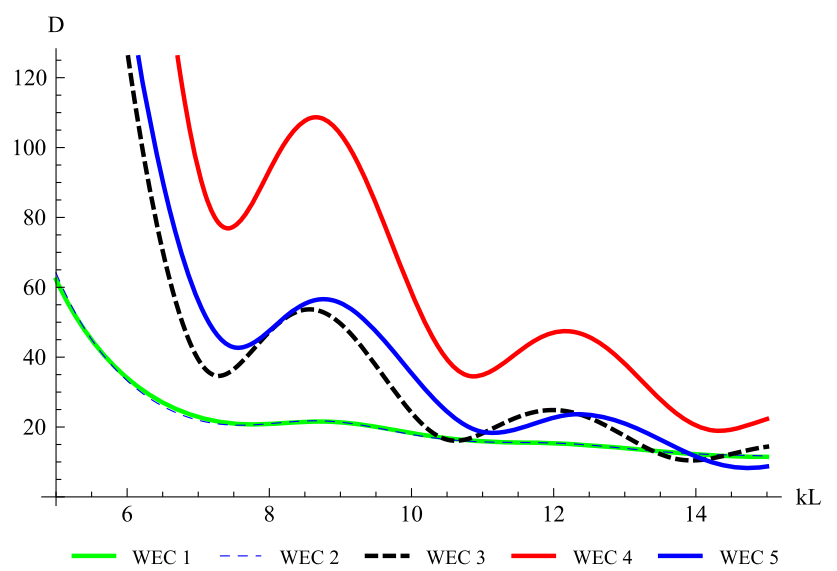

Fig. 7 Predicted optimal displacements of WECs for best linear array optimised for $\beta_{0}=\frac{\pi}{4}$

two and three devices at either end of the array, as shown in Fig. 6.

It can be seen from Fig. 2 that, for $\beta=\frac{\pi}{4}$, this array achieves $q>1$ for the majority of the domain, with the exception when $\mathrm{kL}<7.7$. A maximum value of $q \approx 1.29$ is achieved at $\mathrm{kL} \approx 12.5$, while the minimum value is $q \approx 0.93$ at $\mathrm{kL} \approx 6$. The average performance obtained is $I=1.1431$, which is considerably less than the corresponding optimal array for head seas. Comparing the $\beta$-variation in Fig. 3 with that of the $\beta_{0}=0$ case: there also exists a much smaller range around the optimal value of $\beta=\frac{\pi}{4}$ where $q$ is greater than unity.

The displacements of this optimal array are shown in Fig. 7. Similar to the previous case, large motions of $D>10$ are required for all values of $\mathrm{kL}$, with the general trend that the displacement amplitudes increase for decreasing $\mathrm{kL}$. Although the motions are smaller overall than the optimal $\beta_{0}=0$ case, these displacements are still unacceptably large. This, combined with the more modest performance, suggests this may not be the best choice of array layout.

A similar physical explanation to that in Sect. 3.1 can be applied to the optimal layout in this case. However, due to

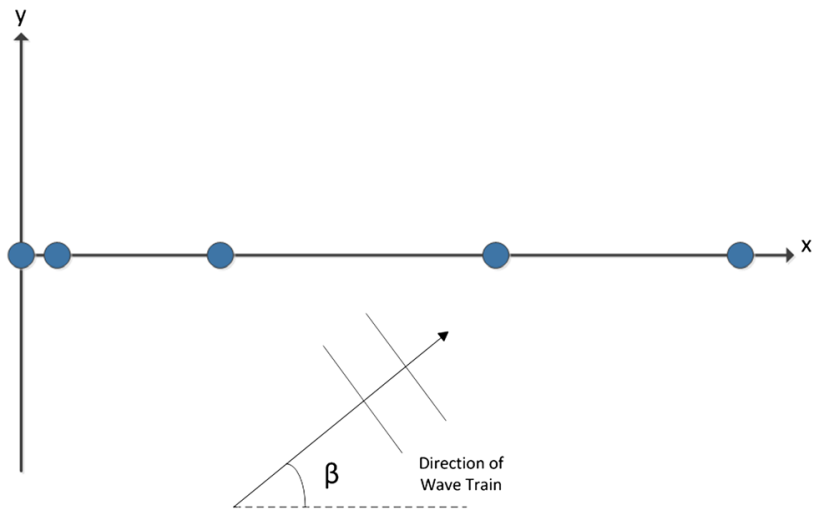

Fig. 8 Diagram of most optimal linear array found for $\beta_{0}=\frac{\pi}{2}$

the intermediate wave angle, the interaction is not as simple, since the wave direction and interaction direction are no longer collinear. More spacing between devices, albeit in two groups at either end of the array, performs better than pushing all devices together. This may be because an increased frontage becomes important for non-head seas and both groups interact well while spanning the length of the array.

\subsection{Beam seas $\beta_{0}=\frac{\pi}{2}$}

The optimisation results for $\beta_{0}=\frac{\pi}{2}$ are shown in Table 4. In this case, only two unique optimal array layouts were discovered by the exhaustive search and the optimisation algorithm. The premier optimal array is shown in Fig. 8, which consists of a group of two devices accompanied by three relatively separated devices. It is interesting that despite the array orientation to the incoming wave, the optimal layout is not symmetric as may have been expected in this case.

Figure 2 shows that at the optimal wave angle ( $\beta=$ $\beta_{0}=\frac{\pi}{2}$ ), this array achieved $q>1$ for the entire range of $\mathrm{kL} \in[5,15]$. An average interaction factor of $I=1.3643$ is achieved along with an impressive maximum of $q \approx 1.7$ around $\mathrm{kL}=13.7$. The interaction factor appears to increase with increasing $\mathrm{kL}$ until it reaches this maximum value, where it begins to decrease with increasing $\mathrm{kL}$. Although this is the highest peak in $q$ achieved by any of the optimal arrays thus far, the average value of $I=1.3643$ falls below that of the best $\beta_{0}=0$ array.

Figure 3 shows that there is a surprisingly large range of approximately $\pm \frac{3 \pi}{16}$ around the optimal value of $\beta=\frac{\pi}{2}$ where $q$ remains greater than unity. Comparing all the curves of $q$ vs $\beta$ for the different optimal arrays, it is clear that the shape of the $q$ vs $\beta$ curve is altered so as to achieve good performance at $\frac{\pi}{2}$, which results in poorer performance at other wave angles. It is interesting to compare the curves in Fig. 3 and note the change in behaviour as different optimal wave angles are considered. As the incident wave angle changes, 


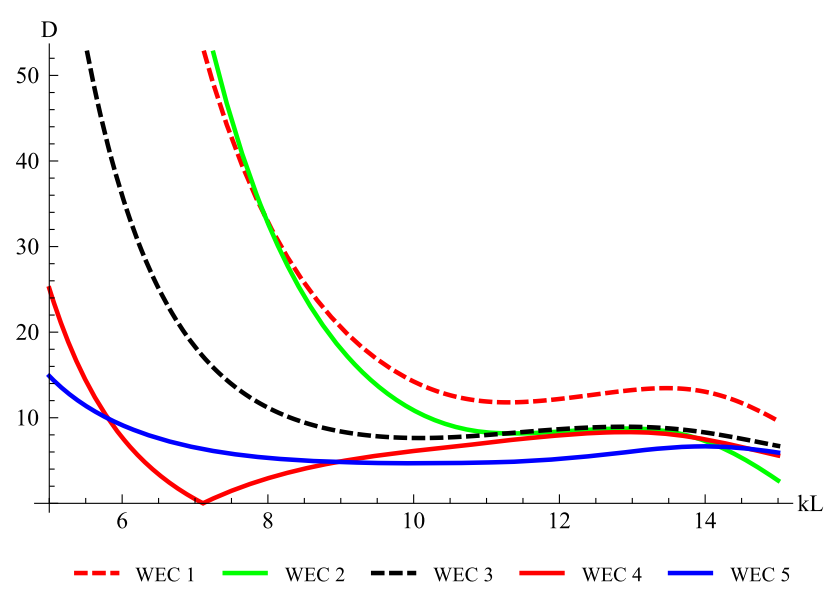

Fig. 9 Predicted optimal displacements of WECs for best linear array optimised for $\beta_{0}=\frac{\pi}{2}$

the plot of $q$ vs $\beta$ alters to increase at the desired value of $\beta=\beta_{0}$; this necessarily results in poorer performance at other angles.

The displacements for this optimal array are shown in Fig. 9. These are unacceptably large $(D>5)$ for the entire domain of $\mathrm{kL} \in[5,15]$ for all but WEC 4 , whose displacements approach zero only in the close vicinity of $\mathrm{kL} \approx 7$. In general, larger displacements are predicted for smaller $\mathrm{kL}$. All devices have $D<20$ for $\mathrm{kL} \in[10,15]$, suggesting that this region may not be as much affected by imposition of motion constraints than the other optimal linear arrays. As expected, the grouped devices exhibit larger motions than the relatively isolated WECs. It is interesting that, in the optimal scenario, WEC 4 has $D=0$ near $\mathrm{kL} \approx 7.1$. The combination of high $q$-factor, large range of $\beta$ stability and relatively low displacements in the region $\mathrm{kL} \in[10,15]$ indicate this array may be an ideal candidate for WEC array design.

Due to the normal wave incidence onto the array (wave direction and interaction direction are perpendicular), it stands to reason that greater frontage would result in better overall performance as opposed to groups of WECs. It may be that groups of devices with large spaces between these groups would not be ideal in this case, as much of the power incident on the spaces may escape through the array. This is a tentative explanation of the more spread-out layout obtained here. However, one closely spaced pair is still present at one side, indicating that uniform spacing is not optimal. It is unclear why this layout is not symmetric; perhaps this is due to the fact that the optimisation is maximising the mean of $q$ over $\mathrm{kL} \in[5,15]$. Another possible explanation is that there exists a symmetric optimum which is very unstable, such that a small change in array parameters destroys this optimum and thus the optimisation failed to converge to it.

\section{Optimisation of circular arrays}

Two cases of circular arrays are considered and compared in this section. The first is an array with six devices constrained to lie on a circle circumference of radius $r$. The second case is an array of $N=7$ devices, with the extra device fixed in the centre of the circle. Clearly, the optimisation of each array involves the same number of variables, although the calculation of the objective function and hence the optimisation is expected to be somewhat longer in the second case. A diagram of the array without a central device is shown in Fig. 10 for clarity.

For comparative purposes, the performance of the uniform versions of these arrays $\left(\theta_{1}=\cdots=\theta_{5}=\frac{\pi}{3}\right)$, both with and without the central WEC are presented. Figures 11 and 12 show the variation of the $q$-factor for the uniform array with changes in $\mathrm{kL}$ and $\beta$, respectively. Table 5 shows the average interaction factor for these arrays for several wave angles. Since the performance of the uniform array is $\frac{\pi}{3}$ periodic and symmetric about multiples of $\frac{\pi}{3}$, only $\beta_{0}=0, \frac{\pi}{6}$ are shown.

Overall, the uniform layouts do not perform well over the entire range of $\mathrm{kL} \in[5,15]$, and performance is very oscillatory for changes in $\beta$. The inclusion of a central device improves performance only in some cases and decreases performance slightly in others. The interaction factor (and its mean) appear to be highly dependent on the incident wave angle for the uniform cases.

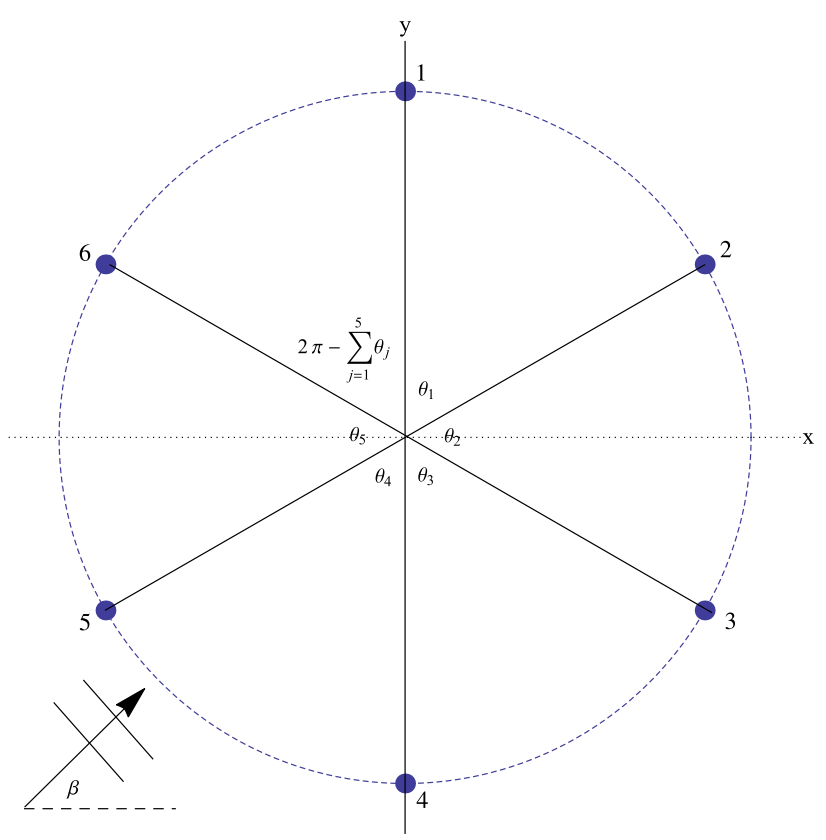

Fig. 10 Diagram of general six-device circular array, without central device 


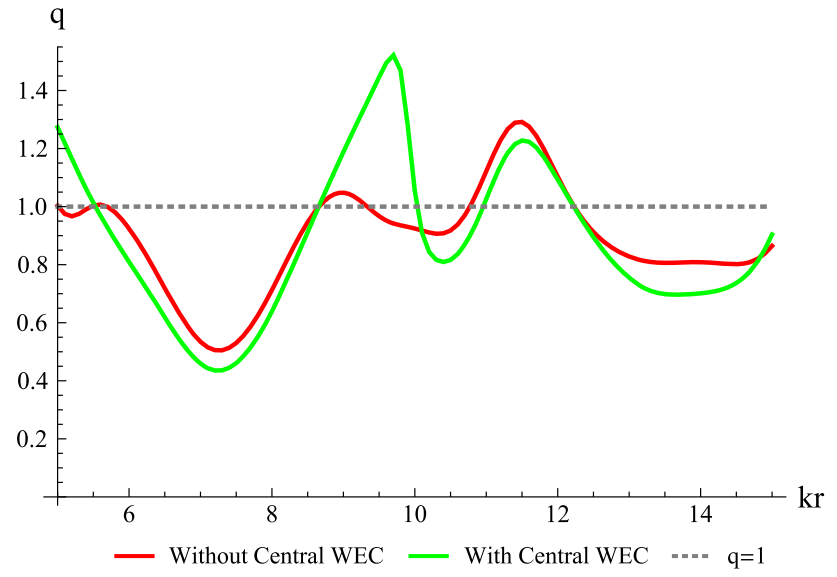

Fig. 11 Interaction factor $q$ against non-dimensional radius kr for uniform six and seven WEC arrays, with $\beta=0$

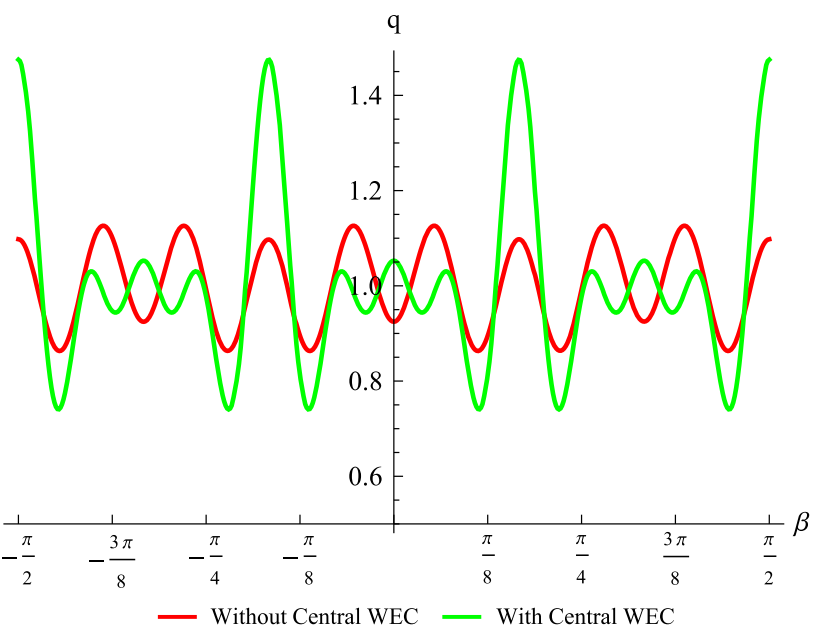

Fig. 12 Interaction factor $q$ against incident wave angle $\beta$ for uniform six and seven WEC arrays, with $\mathrm{kr}=10$

Table 5 Average interaction factors $I$ for uniform circular arrays $\left(\theta_{1}=\right.$ $\cdots=\theta_{5}=\frac{\pi}{3}$ )

\begin{tabular}{lll}
\hline & $\beta_{0}$ & $I$ \\
\hline No central device & 0 & 0.890253 \\
& $\frac{\pi}{6}$ & 1.0654 \\
With central device & 0 & 0.883032 \\
& $\frac{\pi}{6}$ & 1.12195 \\
\hline
\end{tabular}

\subsection{Circular arrays without central device}

The angular position of one device (WEC 1) is fixed and is arbitrarily forced to lie at the top of the array, so $\alpha_{1}=$ $\frac{\pi}{2}$. These arrays have five optimisation variables, namely $\theta_{1}, \ldots, \theta_{5}$, which define the positions of the WECs on the circumference as seen in Fig. 10. The array is optimised as in the previous section about the target value of $\mathrm{kr}=10$, and it is assumed that the sea state in question is such that $\left[\mathrm{kr}_{l}, \mathrm{kr}_{u}\right]=[5,15]$; this is arbitrarily chosen to represent a typical case although the method is applicable for any values. As before, constraints on the device motions are not imposed.

Intuitively, it can be assumed that symmetry exists about $\beta=\frac{\pi}{2}$, since an optimal array found for an angle $\beta=$ $\beta_{0}<\frac{\pi}{2}$ is equivalent to an analogous reflected array with $\beta=\frac{\pi}{2}+\beta_{0}$. Therefore, results are presented only for $\beta_{0} \in$ $\left[0, \frac{\pi}{2}\right]$. The optimisation was conducted in intervals of $\frac{\pi}{8}$; for brevity, detailed results are only shown for intervals of $\frac{\pi}{4}$.

In order to enforce similar spacing restrictions to the previous section, each angular parameter was limited to remain within $0.1 \leq \theta_{j} \leq 2 \pi-0.5$, and the consistency constraint Eq. (18) was also enforced. This minimum bound of $\theta_{j} \geq 0.1$ is slightly more restrictive than that in Sect. 3 , as it corresponds to a minimum separation between consecutive devices of approximately $0.1 \mathrm{kr}$. However, this was found to be necessary to avoid calculation difficulties when calling the objective function, particulary in those cases involving a central device in the array. The upper constraint of $\theta_{j} \leq 2 \pi-0.5$ is to allow the possibility that all but one of the variables are at their minimum allowed value of 0.1 , while retaining consistency.

A search routine similar to the linear array case is run over the search space of possible starting values of the parameters, in an attempt to ensure that the best optima are found. All possible allowed combinations of $\theta_{j}=\left\{\frac{\pi}{5}, \frac{2 \pi}{5}, \ldots \pi\right\}$ for $j=1, \ldots, 6$ were investigated as starting points for the optimisation routine, with some combinations omitted due to consistency considerations. It was noticed for circular array geometries that the objective function was not as well behaved as the linear array case. This is perhaps due to the increased number of variables or the increased complexity of the interaction factor (and hence its mean) due to the array geometry. It is thus acknowledged that the above selection of starting points may not result in the optimisation finding all the possible optimal solutions. Preliminary results show that increasing the number of starting points, by subdividing the range further, results in improvements of optimal values of $I$ of the order of $2-5 \%$. This also results in a considerably longer run time for the optimisation, which renders it unfeasible in the short term. The above set of starting points was chosen as the best practical case. Therefore, unlike Sect. 3, the results presented in this section are not advertised as globally optimum, but rather the best cases found by the optimisation outlined.

The resulting optimal parameter values are presented in Tables 6,7 and 8 for $\beta_{0}=0, \frac{\pi}{4}, \frac{\pi}{2}$. These provide the values of the optimal mean interaction factor $I$ and the optimal layout variables $\theta_{1}, \ldots, \theta_{5}$ in radians, with the remaining angular separation given by (18). The top four cases discovered by the routine are given for each angle of incidence. The best performing arrays for $\beta_{0}=0, \frac{\pi}{4}, \frac{\pi}{2}$ are analysed with 
Table 6 Optimisation results for the six-device circular array (without middle device) with $\beta_{0}=0$

\begin{tabular}{llllll}
\hline$\theta_{1}$ & $\theta_{2}$ & $\theta_{3}$ & $\theta_{4}$ & $\theta_{5}$ & $I_{\mathrm{opt}}$ \\
\hline 0.1 & 1.4707 & 1.4153 & 0.1000 & 3.0972 & 1.5907 \\
0.1 & 0.1000 & 1.0850 & 0.3233 & 0.3529 & 1.5877 \\
0.1 & 1.4669 & 1.4984 & 0.1000 & 1.5424 & 1.5809 \\
0.1 & 1.1992 & 0.3233 & 0.3531 & 4.2076 & 1.5791 \\
\hline
\end{tabular}

Table 7 Optimisation results for the six-device circular array (without middle device) with $\beta_{0}=\frac{\pi}{4}$

\begin{tabular}{llllll}
\hline$\theta_{1}$ & $\theta_{2}$ & $\theta_{3}$ & $\theta_{4}$ & $\theta_{5}$ & $I_{\mathrm{opt}}$ \\
\hline 0.1000 & 0.6512 & 1.5252 & 0.1000 & 0.1000 & 1.5101 \\
2.3500 & 0.1000 & 0.1000 & 1.1174 & 0.3102 & 1.4882 \\
2.2626 & 0.1000 & 0.1000 & 0.1000 & 1.1117 & 1.4516 \\
0.7981 & 1.4109 & 0.1000 & 0.1000 & 0.1000 & 1.4453 \\
\hline
\end{tabular}

Table 8 Optimisation results for the six-device circular array (without middle device) with $\beta_{0}=\frac{\pi}{2}$

\begin{tabular}{llllll}
\hline$\theta_{1}$ & $\theta_{2}$ & $\theta_{3}$ & $\theta_{4}$ & $\theta_{5}$ & $I_{\text {opt }}$ \\
\hline 1.5208 & 0.1000 & 1.5208 & 1.5208 & 0.1000 & 1.5824 \\
0.3097 & 0.8228 & 0.1000 & 3.6801 & 0.1000 & 1.5472 \\
0.2863 & 1.0837 & 0.1000 & 0.1000 & 2.6281 & 1.5309 \\
0.2957 & 1.0793 & 0.1000 & 0.1000 & 0.1000 & 1.5216 \\
\hline
\end{tabular}

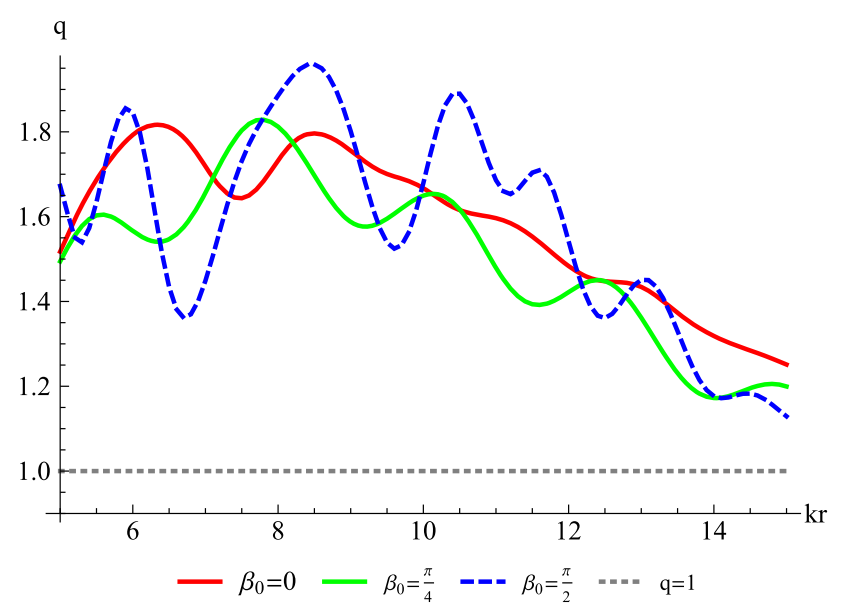

Fig. 13 Interaction factor $q$ against non-dimensional radius $\mathrm{kr}$ for best six WEC circular array for $\beta_{0}=0, \frac{\pi}{4}, \frac{\pi}{2}$

respect to the array layout and optimal displacements in the following subsections. A diagram of the top four layouts is presented for the $\beta_{0}=0$ case. For brevity, only the best array layout is presented for other wave angles. The variation of the interaction factor of all the best arrays with changes in $\mathrm{kr}$ and $\beta$ are shown in Figs. 13 and 14, respectively.

\subsubsection{Angle of incidence $\beta_{0}=0$}

Table 6 shows the optimisation results for the six-device circular array for $\beta_{0}=0$. The best array layouts are shown in Fig. 15. These layouts differ from each other; however, all involve closely spaced groups of at least two devices.

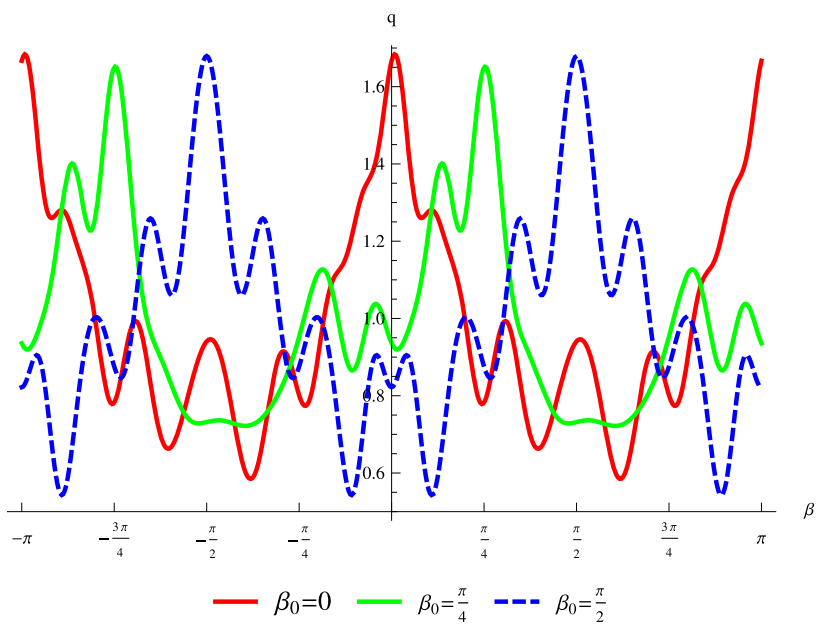

Fig. 14 Interaction factor $q$ against incident wave angle $\beta$ for best six WEC circular array for $\beta_{0}=0, \frac{\pi}{4}, \frac{\pi}{2}$ with $\mathrm{kr}=10$

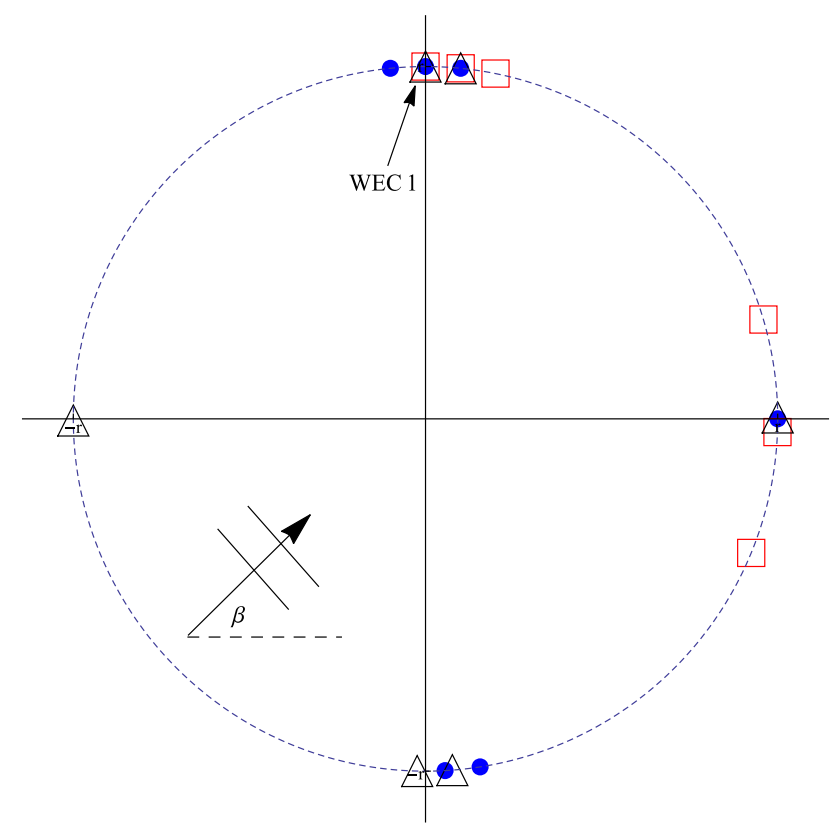

Fig. 15 Diagram of best six-device circular arrays found for $\beta_{0}=0$. The best performing array $(I=1.5907)$ is shown by the solid blue circles, the second best $(I=1.5877)$ by the hollow red squares, and the third best $(I=1.5809)$ by the hollow black triangles. The fourth best $(I=1.5791)$ array is very similar to the second and is omitted for clarity. WEC 1 is marked and WECs $2-6$ are numbered in a clockwise order' (colour figure online) 


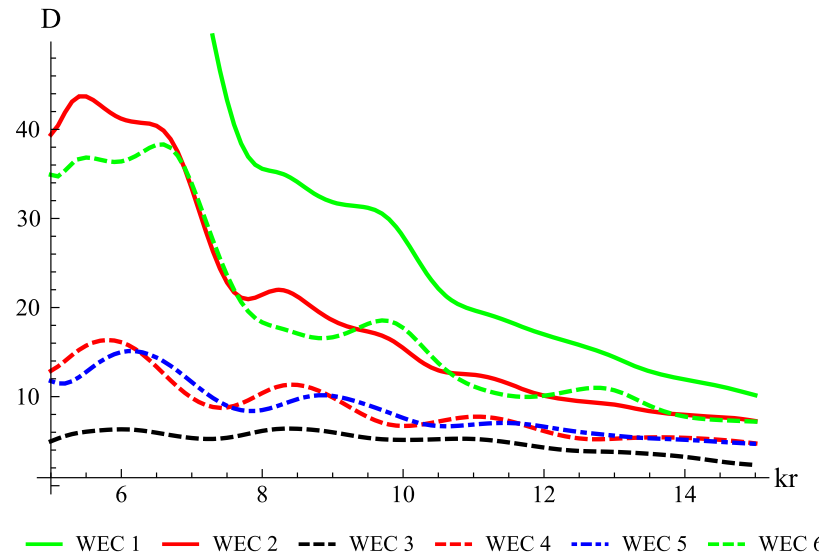

Fig. 16 Optimal displacements for best six-device circular array optimised for $\beta_{0}=0$

The best layout occurs with groups of two and three devices at the bottom and top of the array, respectively, and an isolated device to the right. Note that the devices can almost be considered to be arranged within a semicircle orientated opposite to the incident wave direction. This array achieves an average interaction factor of $I=1.5907$ and Fig. 13 shows that it achieves a peak value of $q \approx 1.8$ around $\mathrm{kr} \approx 6.2$ and $\mathrm{kr} \approx 8.2$. Above these values of $\mathrm{kr}, q$ steadily decreases to the minimum value of $q \approx 1.26$ at $\mathrm{kr}=15$.

Figure 14 shows that there is a relatively large range of $\pm \frac{\pi}{5}$ approximately about $\beta=0$, where $q$ remains greater than unity. Away from this range, it is also evident that quite poor performance occurs at other wave angles and which would be expected.

The non-dimensional displacements of each device in this array are shown in Fig. 16. In keeping with previous results, the grouped devices exhibit relatively large motions compared with the isolated device. The displacement of the isolated device (WEC 3 ) is maintained around $D \approx 5$ for the entire domain of kr. All other devices have $D>6$ for all $\mathrm{kr} \in[5,15]$, with the group of three devices at the top of the array (WECs 1,2 and 6) having considerably larger displacements, especially for lower kr.

A similar physical interpretation of the optimal layout to Sects. 3.2 and 3.3 may apply here, with the two groups interacting well at either end of the array, with the WEC 3 capturing power incident through the middle of the array. The semicircular arrangement may be due to a need to avoid "rows" of devices, i.e. devices stacked behind one another, unless these device are within an interacting group.

\subsubsection{Angle of incidence $\beta_{0}=\frac{\pi}{4}$}

The optimisation results for $\beta_{0}=\frac{\pi}{4}$ are presented in Table 7 and a diagram of the best layout in this case is presented in Fig. 17. This exhibits similar features to the previous array,

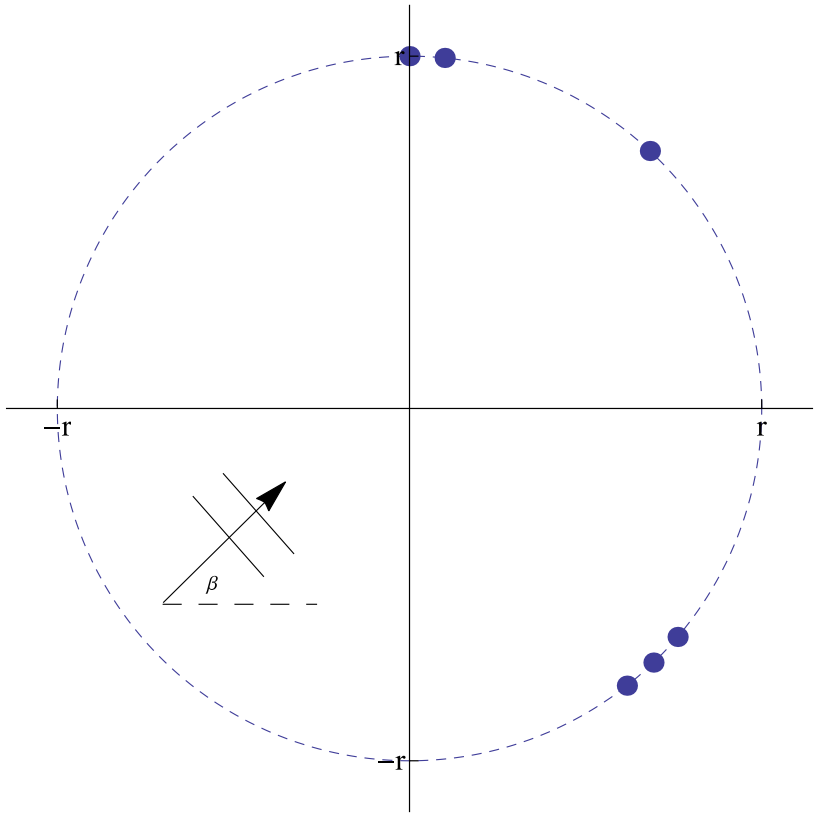

Fig. 17 Diagram of best six-device circular array found for $\beta_{0}=\frac{\pi}{4}$

with groups of two and three WECs and a relatively isolated device between them. Again, all the WECs lie within a semicircle that is almost opposite the incident wave direction.

This array achieves a mean interaction factor of $I=$ 1.5101 and maximum value of $q \approx 1.8$ near $\mathrm{kr} \approx 7.7$, as seen in Fig. 13. The overall behaviour of $q$ is more oscillatory than the optimal $\beta_{0}=0$ case and results in a reduced average value. From Fig. 14, the behaviour of this array for variation in $\beta$ is not dissimilar to the $\beta_{0}=0$ case, except that the peak value is centred around $\beta=\frac{\pi}{4}$, instead of $\beta=0$.

A broadly similar behaviour of the WEC displacements is also observed in Fig. 18. Once more the isolated device (WEC 3 ) has the smallest motion amplitudes of $D \approx 5$ for the entire range, and the grouped devices exhibit larger motions, especially for the group of three devices (WECs 4, 5 and 6 ). This behaviour is most evident for smaller kr, with the relative differences decreasing for larger kr. For kr $>9$ the isolated device and the group of two devices (WECs 1 and 2) have very similar motions.

A similar physical explanation to the previous array in Sect. 4.1.1 can be applied to this array layout, with two groups at either end of the array, with respect to the incident wave direction, and a single WEC bridging the gap between these groups. The performance of this array is less than that in Sect. 4.1.1, presumably due to the fixed position of WEC 1 and the associated lack of freedom.

\subsubsection{Angle of incidence $\beta_{0}=\frac{\pi}{2}$}

Table 8 shows the optimisation output for $\beta_{0}=\frac{\pi}{2}$. Figure 19 shows that the best array discovered is symmetric, with 


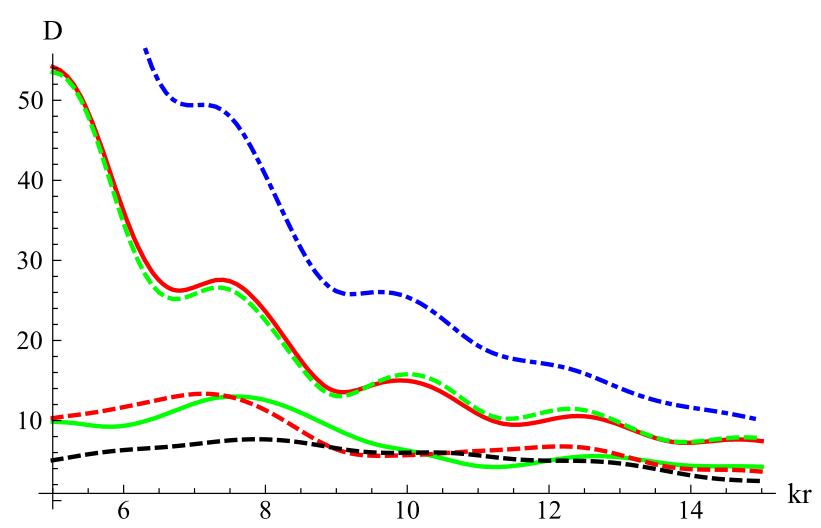

— WEC 1 --- WEC 2 --- WEC 3 - WEC 4 --. WEC 5 --- WEC 6

Fig. 18 Optimal displacements for best six-device circular array optimised for $\beta_{0}=\frac{\pi}{4}$

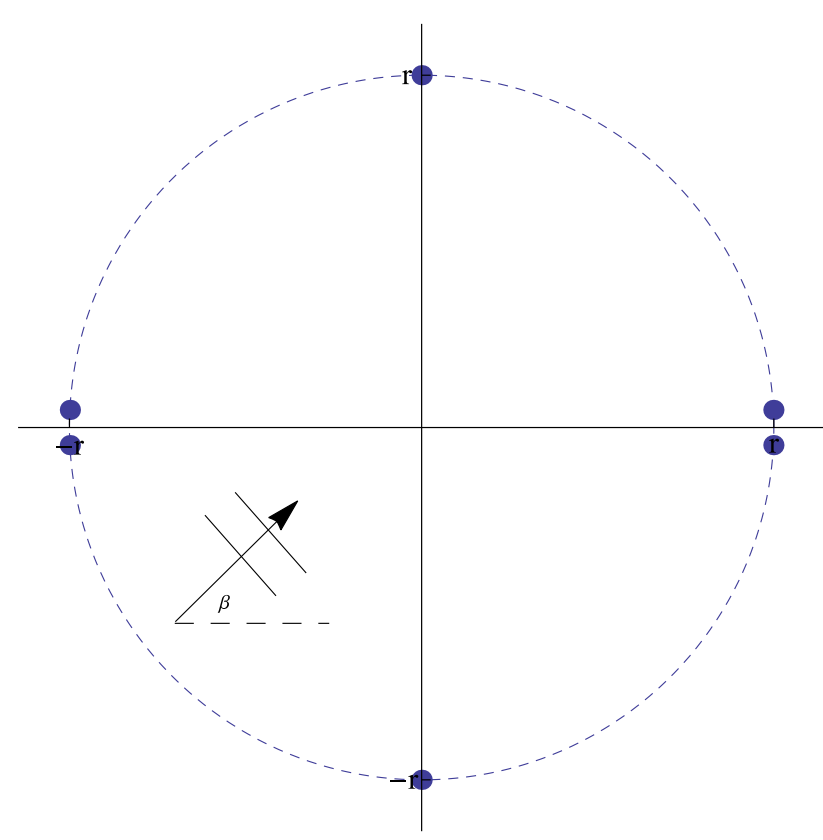

Fig. 19 Diagram of best six-device circular array found for $\beta_{0}=\frac{\pi}{2}$

groups of two devices at the left and right and with two isolated devices at the top and bottom. It is interesting that, despite not enforcing symmetry, the optimisation converged to a symmetric array layout.

The average interaction factor achieved by this array is $I=$ 1.5824 , which is similar to the $\beta_{0}=0$ case, although slightly lower. The maximum value of $q \approx 1.96$ attained at $\mathrm{kr} \approx$ 8.5 is considerably higher than previous arrays. The overall behaviour of $q$ is more oscillatory than the other arrays of this type, as seen in Fig. 13. This may be undesirable as it would cause variability in performance over the range of $\mathrm{kr}$. For the $\beta$-variation, a similar performance to the $\beta_{0}=0$ case is seen for this array in Fig. 14, with the performance peak centred instead at $\beta=\frac{\pi}{2}$.

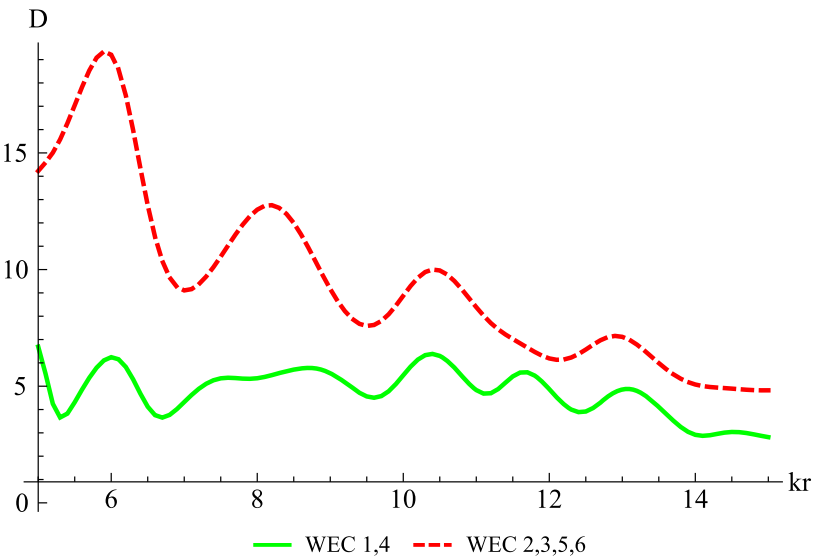

Fig. 20 Optimal displacements for best six-device circular array optimised for $\beta_{0}=\frac{\pi}{2}$

The corresponding optimal motions are given in Fig. 20. Due to symmetry, WECs 1 and 4 (isolated) and WECs 2, 3, 5 and 6 (pairs) exhibit the same motions. As with other arrays, the isolated devices maintain $D \approx 5$ for all $\mathrm{kr}$, while the pairs have larger motions, particularly for smaller kr. The displacement amplitudes of the pairs are within [5, 20], which is less than the largest motions for other arrays. This suggests that the imposition of motion constraints may not be as detrimental to this array.

This optimal array layout is a special case, since the fixed device (WEC 1 ) is in line with the incoming wave direction. This is likely why a symmetric optimal layout was converged to here, while in other cases this is less likely due to the fixed position of WEC 1 at the top of the array. Also, similar physical arguments to previous arrays can be applied here, with groups at either side of the array and isolated devices in the middle. In this case, the distance between these central devices (WECs 1 and 4, along the $y$ axis) is $2 \mathrm{kr} \in[10,30]$. Therefore, interaction would be small between these devices and the argument against stacked devices or rows would not apply.

\subsection{Circular arrays with central device}

The configuration of the last section is extended to a circular array of seven devices, with the additional device in the centre of the circle. In this way, one device is fixed at the origin, which is denoted as WEC 7 with $\left(d_{7}, \alpha_{7}\right)=(0,0)$, and the angular position of one device (WEC 1 ) is fixed at the top of the array with $\alpha_{1}=\frac{\pi}{2}$. The number of optimisation variables is, therefore, the same as the previous case and the process follows in a similar manner to Sect. 4.1, with all other optimisation parameters the same.

Optimal parameter values are presented in tabular form for $\beta_{0}=0, \frac{\pi}{4}, \frac{\pi}{2}$ in Tables 9,10 and 11 , respectively. The behaviour of the interaction factor is shown for variation 
Table 9 Optimisation results for the seven-device circular array (including a middle device) with $\beta_{0}=0$

\begin{tabular}{llllll}
\hline$\theta_{1}$ & $\theta_{2}$ & $\theta_{3}$ & $\theta_{4}$ & $\theta_{5}$ & $I_{\mathrm{opt}}$ \\
\hline 0.1000 & 0.1000 & 2.8284 & 0.1000 & 0.1000 & 1.5408 \\
0.1000 & 1.4838 & 1.2789 & 0.1000 & 3.2204 & 1.5366 \\
0.1000 & 1.4738 & 1.4706 & 0.1000 & 0.1000 & 1.5146 \\
1.6028 & 1.4005 & 0.1000 & 0.1000 & 2.9799 & 1.5067 \\
\hline
\end{tabular}

Table 10 Optimisation results for the seven-device circular array (including a middle device) with $\beta_{0}=\frac{\pi}{4}$

\begin{tabular}{llllll}
\hline$\theta_{1}$ & $\theta_{2}$ & $\theta_{3}$ & $\theta_{4}$ & $\theta_{5}$ & $I_{\text {opt }}$ \\
\hline 0.7534 & 1.4852 & 0.1000 & 0.1000 & 3.702 & 1.4957 \\
0.7891 & 1.4185 & 0.1000 & 0.1000 & 0.1000 & 1.4264 \\
0.8021 & 1.4239 & 0.1000 & 0.1000 & 2.8824 & 1.4184 \\
0.1000 & 0.6148 & 0.3467 & 1.0625 & 0.1000 & 1.4024 \\
\hline
\end{tabular}

Table 11 Optimisation results for the seven-device circular array (including a middle device) with $\beta_{0}=\frac{\pi}{2}$

\begin{tabular}{llllll}
\hline$\theta_{1}$ & $\theta_{2}$ & $\theta_{3}$ & $\theta_{4}$ & $\theta_{5}$ & $I_{\text {opt }}$ \\
\hline 1.3060 & 0.1000 & 3.2065 & 0.1000 & 0.1000 & 1.5361 \\
1.4453 & 0.1000 & 0.1000 & 2.8821 & 0.1000 & 1.5055 \\
0.3020 & 0.8243 & 0.1000 & 3.8186 & 0.1000 & 1.4677 \\
1.3198 & 1.9006 & 1.3732 & 0.1000 & 0.1000 & 1.4528 \\
\hline
\end{tabular}

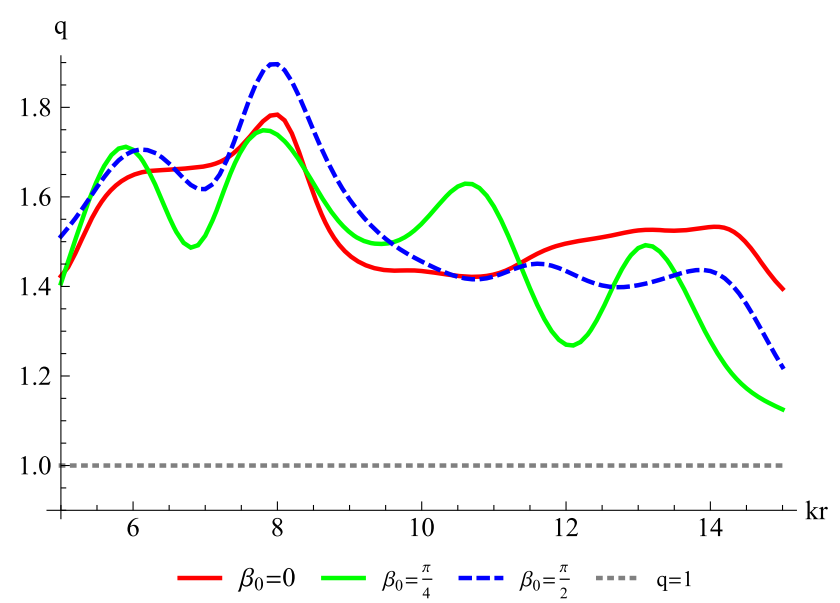

Fig. 21 Interaction factor $q$ against non-dimensional radius $\mathrm{kr}$ for best seven WEC circular arrays for $\beta_{0}=0, \frac{\pi}{4}, \frac{\pi}{2}$

in $\mathrm{kr}$ in Fig. 21 and for variation in $\beta$ in Fig. 22. Diagrams of the best array layouts and plots of the predicted optimal displacements are also provided for each case. The top four layouts are shown for the $\beta_{0}=0$ case while, for brevity, only the best array is presented for other angles of incidence.

\subsubsection{Angle of incidence $\beta_{0}=0$}

Table 9 shows the optimisation results for the seven-device circular array with $\beta_{0}=0$ and the top four array layouts are presented in Fig. 23. As in previous cases, although these arrays differ from each other, all contain groups of two and/or three devices. The second and third best arrays are nearly

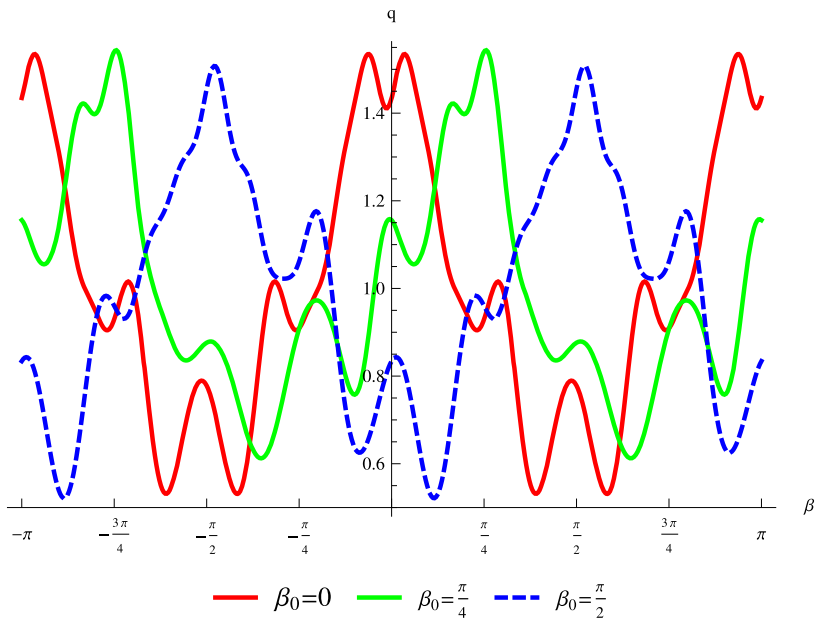

Fig. 22 Interaction factor $q$ against incident wave angle $\beta$ for best seven WEC circular arrays for $\beta_{0}=0, \frac{\pi}{4}, \frac{\pi}{2}$ with $\mathrm{kr}=10$

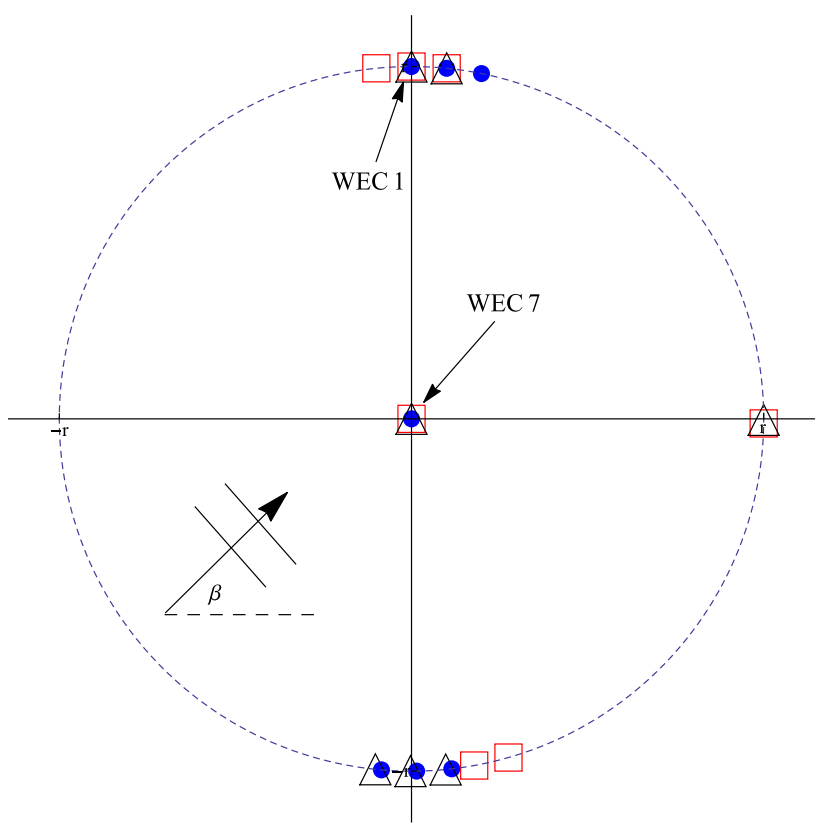

Fig. 23 Diagram of best seven-device circular arrays found for $\beta_{0}=0$. The best performing array $(I=1.5408)$ is shown by the solid blue circles, the second best $(I=1.5366)$ by the hollow red squares, and the third best $(I=1.5146)$ by the hollow black triangles. The fourth best $(I=1.5067)$ array is very similar to the third and is omitted for clarity. WECs 1 and 7 are marked and WECs 2-6 are numbered in a clockwise order (colour figure online) 
mirror images of each other about the $x$ axis; hence their similar performance is due to (12).

The premier array is an almost symmetric layout, with groups of three devices at the top and bottom of the array. Once more, the array members are almost contained within a semicircle opposite the incident wave direction. This array achieves an average interaction factor of $I=1.5408$, with a maximum of $q \approx 1.8$ around $\mathrm{kr} \approx 8$. Overall, the behaviour of $q$ is quite stable, with $q \in[1.4,1.8]$; the sharpest change in $q$ is found around the maximum, where $q$ drops from the maximum value to $q=1.43$ for a unit change in kr. As with most other optimal arrays, Fig. 22 shows that $q$ remains greater than unity for a change of approximately $\pm \frac{\pi}{5}$ in incident wave angle around the optimal angle $\beta_{0}=0$. Also, a double peak can be seen around this optimal value, so for a small change in $\beta$, a small increase in $q$ can in fact be achieved. This also results in a wider peak performance with respect to local $\beta$ variation around $\beta_{0}=0$.

As with previous results, the displacements are unacceptably large for the grouped devices, while the isolated device (WEC 7) exhibits reasonable motions, see Fig. 24. Again for grouped devices, $D>10$ for all $\mathrm{kr}$ considered, with $D$ generally increasing for decreasing $\mathrm{kr}$.

A similar physical interpretation to that in Sect. 4.1.1 can be applied to the optimal layout presented here, since these layouts are quite similar. In this case, WEC 7 is forced to be in the centre of the array so that another WEC is not needed along the $x$ axis to absorb power in the gap between the groups. This results in the two groups containing three devices, presumably to maximise the constructive interference. It should be noted that although the best array presented here has a slightly lower value of $I$ than that in Sect. 4.1.1, it is likely that more power is absorbed by this array due to the extra device.

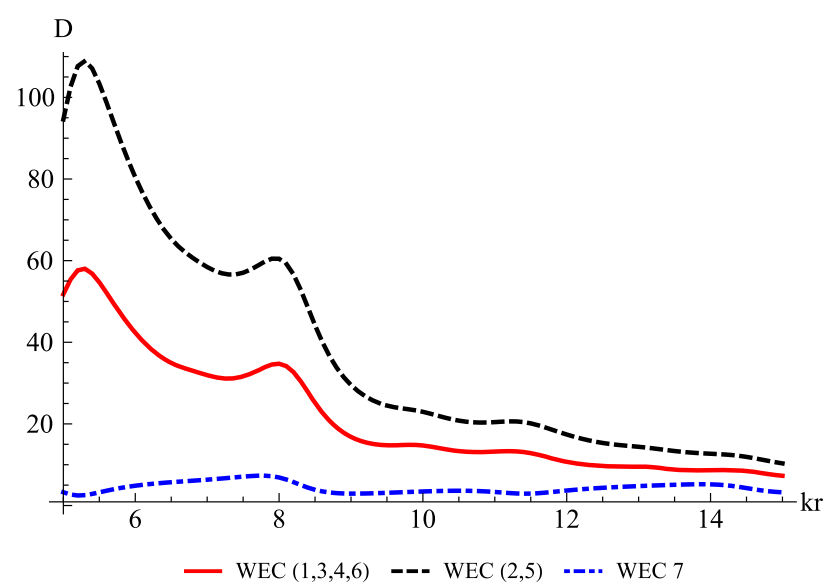

Fig. 24 Optimal displacements for best seven-device circular array optimised for $\beta_{0}=0$. The curves for WEC numbers in brackets are very similar and are omitted for clarity

\subsubsection{Angle of incidence $\beta_{0}=\frac{\pi}{4}$}

The top four array layouts discovered by the optimisation routine are presented in Table 10, and a diagram of the best array is given in Fig. 25. This layout is very similar to the corresponding array in Sect. 4.1.2, with groups of two and three devices along with a relatively isolated device between these groups. Similarly, this array is also positioned within a semicircle opposite the incident wave direction. The average interaction factor of $I=1.4957$ is slightly lower than the corresponding six-WEC array and considerably lower that the seven WEC $\beta_{0}=0$ case.

Figure 21 shows that the variation of $q$ with $\mathrm{kr}$ is more oscillatory than the other arrays. A larger than average range of $\pm \frac{3 \pi}{16}$ for $\beta$-variation around $\beta_{0}=\frac{\pi}{4}$ exists, where $q>1$ for this array. The optimal device motions, shown in Fig. 26, are unacceptably large $(D>5)$ for most devices, particularly the group of three devices (WECs 3, 4 and 5). The central device has $D \approx 4-5$ for all $\mathrm{kr} \in[5,15]$ and similarly the paired (WECs 1 and 6) and isolated device (WEC 2) have $D \approx 6-10$ for the entire range.

A similar physical explanation to previous arrays is given for the best layout presented here. Again, the two groups at the sides of the array presumably maximise constructive interference by the radiated wave field. WECs 2 and 7 may be located in the middle of the array to absorb the power that would otherwise escape through the gap between these groups.

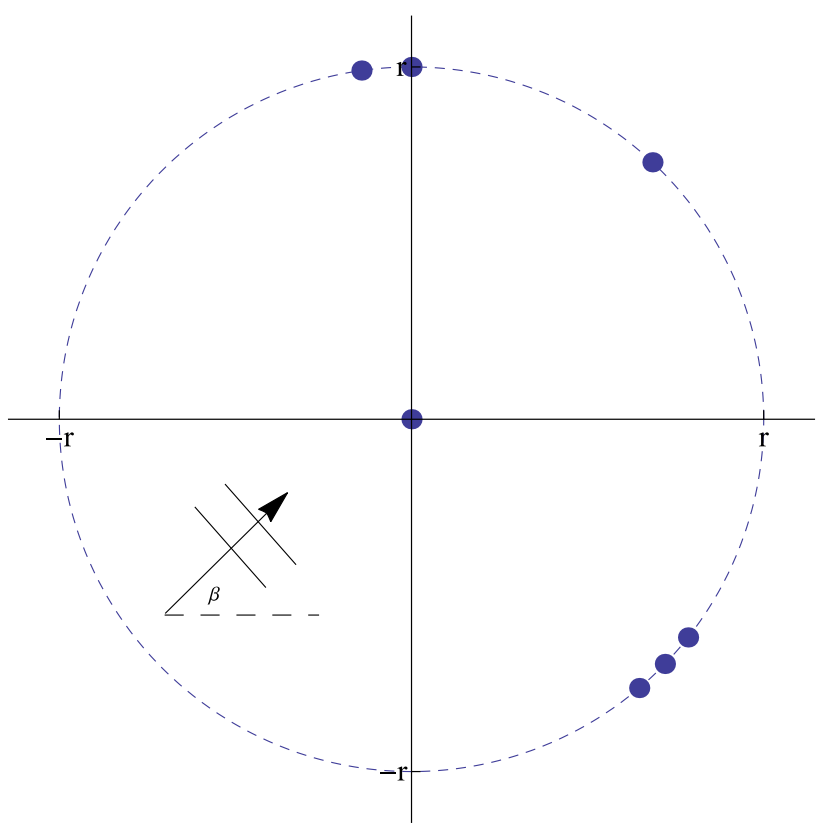

Fig. 25 Diagram of best seven-device circular array found for $\beta_{0}=\frac{\pi}{4}$ 


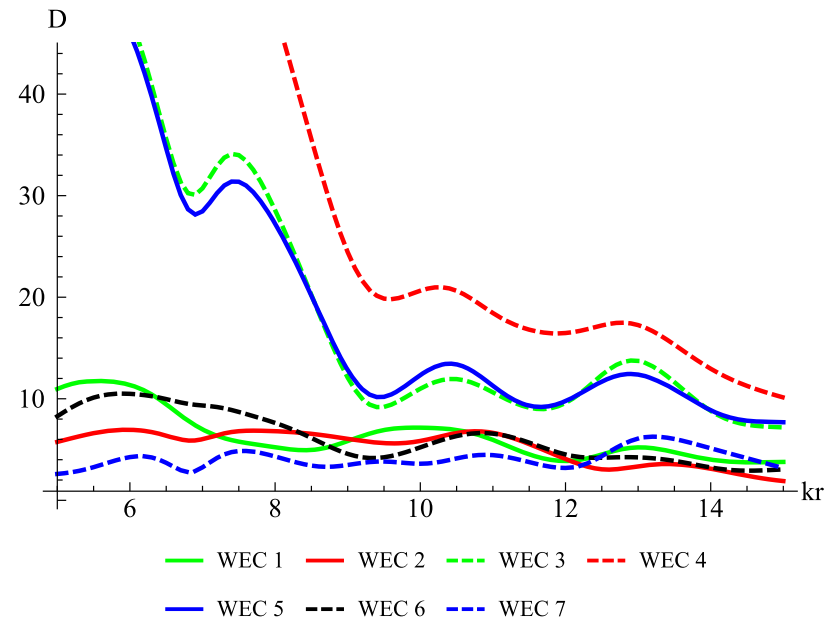

Fig. 26 Optimal displacements for best seven-device circular array optimised for $\beta_{0}=\frac{\pi}{4}$

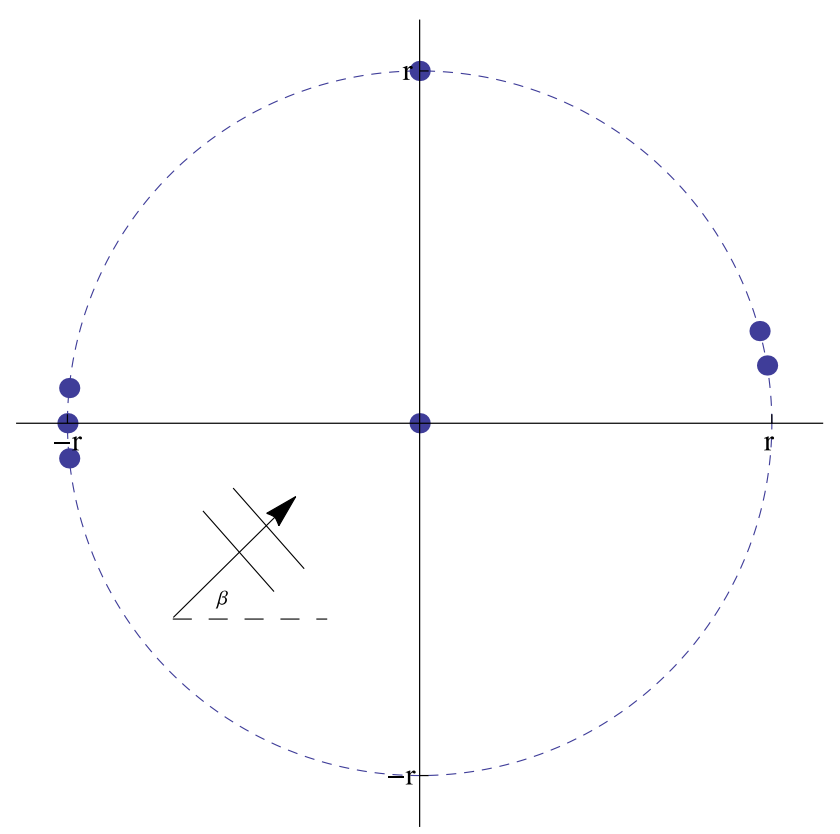

Fig. 27 Diagram of best seven-device circular array found for $\beta_{0}=\frac{\pi}{2}$

\subsubsection{Angle of incidence $\beta_{0}=\frac{\pi}{2}$}

Table 11 shows the optimisation results for $\beta_{0}=\frac{\pi}{2}$ and the best array layout is shown in Fig. 27. Again, the familiar pattern of groups of two and three devices with an isolated device between them can be recognised, almost lying in a semicircle opposite the incident wave direction. The average performance of this array is $I=1.5361$, and Fig. 21 shows that a maximum of $q=1.9$ is achieved around $\mathrm{kr}=8$. The overall performance within $\mathrm{kr} \in[5,15]$ is relatively stable, except near this maximum where $q$ drops to 1.4 as $\mathrm{kr}$ approaches 10 and towards the upper bound of the range, where $q$ drops to less than 1.25 .

Figure 22 shows a large range of $\pm \frac{\pi}{4}$ in $\beta$-variation where constructive interference remains present. This is due to an offset double peak in $q$ vs $\beta$. The array necessarily performs very poorly for other wave angles away from $\beta_{0}=\frac{\pi}{2}$, where the lowest value attainted is $q \approx 0.51$.

Figure 28 shows that the central device exhibits relatively reasonable motions for certain values of $\mathrm{kr}$, but in general most devices have $D>5$ for the majority, if not all, of $\mathrm{kr} \in[5,15]$. As with previous results, optimal displacements are shown to be very large for the group of three devices, particularly for smaller kr. The paired devices have somewhat smaller motions, but are still unacceptably large.

The physical justification behind the optimal layout here is similar to that in the previous section. The array contains two groups at either end of the array to maximise interference, while two devices are present in the centre of the array (along the $y$ axis) to bridge the gap between these groups. It is unclear why the best layout found here is not symmetric, analogous to that in Sect. 4.1.3. This may be because the optimisation did not converge to a global optimum, due to the relatively small range of starting points chosen to allow the optimisation to run in a reasonable time, as mentioned.

\section{Discussion and conclusion}

This paper describes an optimisation method for arrays of five to seven WECs, constrained to linear or circular geometries, such that the optimal array layouts are stable to changes in non-dimensional parameters. This is an extension of previous work, which did not necessarily optimise arrays with the explicit examination of stability. It has been shown here that it is possible to obtain arrays that perform well over a relatively large range of non-dimensional length or radius $(\mathrm{kL} \in[5,15]$ or $\mathrm{kr} \in[5,15])$ for fixed incident wave angle. The work of McGuinness and Thomas (2015) has been extended to more general linear arrays without enforced symmetry and is extended further to examine circular array geometries.

Prior to the present work, the array layout parameters were optimised with respect to the interaction factor, which often resulted in a large peak value of $q$, when constructive interference is achieved but this peak was often surrounded by large troughs of destructive interference. By optimising with respect to the mean of the interaction factor over a certain non-dimensional length, layouts are obtained which perform well on average in this range and not just at a specific value. In most cases, the optimal arrays achieved $q>1$ for the entire range of non-dimensional length/radius considered. It has also been shown that this performance was maintained for large ranges of incident wave angle of up to $\beta_{0} \pm \frac{\pi}{5}$, 


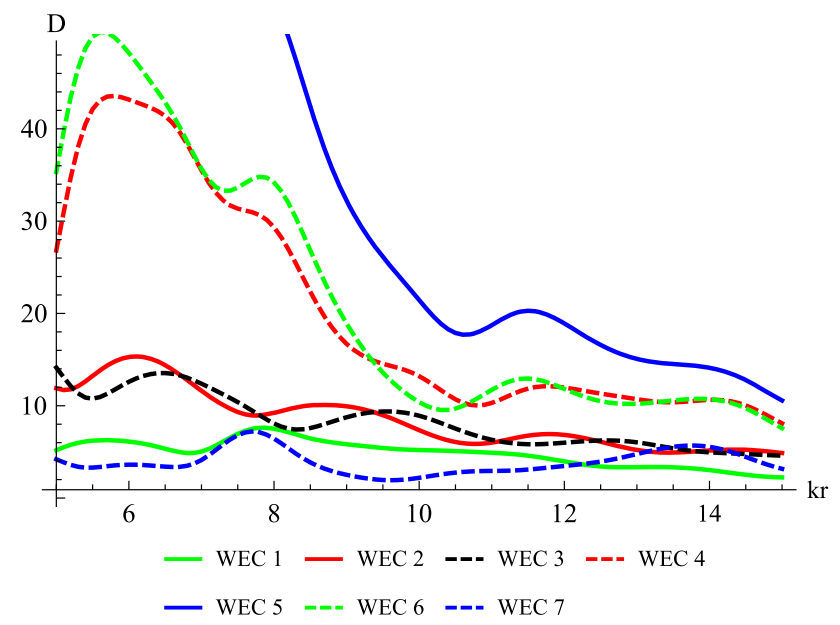

Fig. 28 Optimal displacements for best seven-device circular array optimised for $\beta_{0}=\frac{\pi}{2}$

so that a small change in $\beta$ does not result in destructive interference between the array members.

It should be stressed that the results presented in this paper are valid only for $\mathrm{ka}=0.4, \mathrm{~kL} \in[5,15]$ or $\mathrm{kr} \in[5,15]$, $\beta=\beta_{0}$ and optimal hydrodynamic motions. Movement away from these parameter values or ranges would likely result in poorer performance, particularly for large changes in $\beta$. The imposition of motion constraints and the resulting non-optimal motions would also have a considerable effect on performance. Power take-off is also assumed ideal and inefficiencies are not accounted for.

One major problem with the optimal arrays obtained in this work is the magnitude of the associated device motions. Previous research, such as Thomas and Evans (1981) and Fitzgerald (2006), suggest that an upper limit of device motions should be at most three times the incident wave amplitude. This limit was exceeded by the majority of WECs in all the optimal arrays discovered. These motions are predicted using linear wave theory, which assumes all motions are at most of the same order as the wave motion and is assumed to be small in some sense. Large device motions violate this approximation and thus invalidate the underlying linear wave theory. Therefore, nonlinear effects will tend to damp these large motions, which in turn will affect device interaction and hence array performance.

In general, better performing arrays are expected to involve larger device motions. In some cases presented here, better performance was achieved relative to other optimal arrays, despite smaller predicted device motions. This may indicate that the imposition of device motion constraints may not be as limiting in these cases and it is possible that good performance can be maintained, though this is yet to be confirmed.
Many optimal array layouts presented here include closely spaced groups of two, three or four devices. These groups of WECs are predicted to exhibit the largest motions, with larger motion amplitudes for larger groups. Placing WECs in such close proximity identifies other possible difficulties, such as collisions between devices. In addition, the point absorber approximation may no longer be valid in this case, as it may be unreasonable to neglect the scattered wave field if the WECs are in such close proximity to each other.

One possible solution to this problem may be to replace these groups of devices with an appropriately sized larger device. These arrays would then contain devices of different sizes, a possibility previous research seems to have neglected. These arrays could then be considered as "satellite" arrays, since one could often describe such arrays as involving several smaller devices "orbiting" a larger device. This idea was initially suggested by McGuinness and Thomas (2015), where it was mentioned that larger devices did indeed reduce device motions. It is not known what the effect on power absorption and array performance would be. Another possibility would be to replace these groups of devices with a single device that absorbs power (oscillates) in two modes of motion, thereby recreating the dipole effect often exhibited by the pairs of devices in optimal layouts. It is hoped that either a larger device or one that operates in two (or more) modes would recreate the constructive interference pattern without the issues of close deployment and large motions.

The results for linear arrays in this paper agree with those of McGuinness and Thomas (2015), although these are improved upon due to the increased generality of this work. As mentioned previously, other values of $\beta_{0}$ were investigated but omitted here for brevity. Similar to McGuinness and Thomas (2015), it was found that for $\beta_{0}=\frac{\pi}{8}$, in the general case considered here, linear arrays performed poorly with $I<0.9$ in even the optimal case. This suggests that this wave angle should be avoided.

In general, this work shows that optimal six and seven WEC circular arrays can perform better than optimal five WEC linear arrays, as seen by the higher values of $I$ obtained in Sect. 4. This may be due to the enforced geometry performing better in the circular case, or it may be due to the increased freedom in the optimisation due to the larger number of variables. It should also be noted that the optimisation calculation took considerably longer time to execute for the circular arrays and the arrays found are not guaranteed to be globally optimal, as mentioned in Sect. 4. Again, this was likely due to a combination of increase in complexity of the objective function due to the geometry or to the increased number of variables.

As mentioned in Sect. 4, a search of the variable space for starting points of the optimisation was conducted; however, it was found that a prohibitively long run time resulted when an exhaustive search was performed. Therefore, a more sparse 
search of the variable space was conducted, which produced results in a reasonable time. It was acknowledged that the results presented pertaining to circular arrays are not globally optimum. A preliminary comparison indicated that a $2-5 \%$ improvement can be achieved for a more exhaustive search, with a 20- to 50-fold increase in run time.

As mentioned, symmetry would be expected in the optimal arrays about $\beta_{0}=\frac{\pi}{2}$. However, this was not achieved for the optimisations performed. This was due to the optimisation not finding a global optimum and converging to a local maximum which was not necessarily analogous to the maximum found for the corresponding symmetric wave angle. It was confirmed that symmetry was achieved for optimal arrays with $\beta_{0}=0, \frac{\pi}{2}$ and $\beta=\frac{\pi}{4}, \frac{3 \pi}{4}$ when an exhaustive search was preformed.

Comparing the results of Sects. 4.1 and 4.2, it is clear that the addition of the seventh device in the centre of the circle influences the array performance and optimal array layout, even thought this does not add any extra variables to the optimisation. The results shown here, particularly the optimal values of $I$, suggest that the inclusion of an extra device reduces the average optimum interaction factor. This is contrary to expectation, as preliminary results by C. Costigan (Modelling Circular Arrays of Wave-Power Device, Project Report, University College Cork, 2014), on similar symmetric arrays of one variable, suggested the opposite. This work optimised circular arrays with different (a)symmetries imposed, so that each array is described by just one variable. The arrays were optimised by maximising the mean of the interaction factor over the non-dimensional radius. It may be that, due to the strict assumptions of array layouts (symmetry, one variable etc.), the inclusion of a central device improved performance in that special case and that this does not hold for a more general layout. The work of Costigan also showed that the optimal arrays tended to contain groups of devices, albeit in a simpler optimisation regime. This agrees with the results presented here and with those of McGuinness and Thomas (2015).

For the circular arrays presented here, it was noted that the arrays often converged to a layout that was approximately contained in a semicircle facing in the same direction as the incident wave angle. It is interesting that optimal interaction and constructive interference seem to occur when all devices are located in a semicircle, the orientation of which is dependent on the incident wave angle. Future work will examine this effect further and investigate the possibility of limiting an optimisation to a semicircle, which would be computationally more efficient.

The best average interaction factor was achieved for the six-device circular array, i.e. without middle device, with $\beta_{0}=0$, where $I=1.5907$ was obtained. The behaviour of the interaction factor was relatively stable to changes in both $\mathrm{kr}$ and $\beta$. However, very large displacements were pre- dicted in the optimal case, with $D>5$ for most devices and $D \in[10,75]$ for the group of three devices. The best overall array discovered within this work, which had a balance between good performance and relatively small motions, was the optimal linear array for $\beta_{0}=\frac{\pi}{2}$, as discussed in Sect. 3.3. Within the region of $\mathrm{kL} \in[10,15]$, this array achieves $q \in[1.3,1.7]$, while the non-dimensional displacement of all the array members are $D \in[5,15]$. These displacements are considerably lower than WECs within other optimal arrays. Since a motion constraint would have a lesser relative effect on these motions, it is reasonable to suggest that this constraint would also have a less relative impact on array performance, though this has yet to be confirmed and will be examined in future work. The array also has a large range (approximately $\pm \frac{3 \pi}{16}$ ) of $\beta$ variability, around the optimal value of $\beta_{0}=\frac{\pi}{2}$, where $q>1$ is maintained.

Future research in this area will consider device motion constraints and assess the effect on array performance and optimal array layout. This will be done by including the device displacements as variables in the objective function, with upper limits imposed on the amplitudes. It is also planned to examine other array geometries, such as triangular and elliptical layouts. Note that in order to define the mean of the interaction factor over some non-dimensional length, it is necessary to define an array geometry as the mean interaction factor has no sensible definition for general two-dimensional geometries. Further investigation into semicircular array layouts will also be conducted and extended to semi-elliptical arrays, as results in this paper suggest that this may be the optimal case when devices are constrained to such geometries. Investigation of an alternate objective function, where the average is taken over a small range of incident wave angle, instead of non-dimensional array length/size, is also planned.

\section{References}

Child BFM (2011) On the configuration of arrays of floating wave energy converters. Ph.D. thesis, The University of Edinburgh

Evans DV (1979) Some theoretical aspects of three-dimensional waveenergy absorbers. In: First Symposium on Ocean Wave Energy Utilization. Gothenburg, Sweden

Falnes J (1980) Radiation impedance matrix and optimum power absorption for interacting oscillators in surface waves. Appl Ocean Res 2(2):75-80

Fitzgerald C (2006) Optimal configurations of arrays of wave power devices. Master's thesis, University College Cork

Fitzgerald C, Thomas GP (2007) A preliminary study on the optimal formation of an array of wave power devices. In: Seventh European Wave and Tidal Energy Conferance. Porto, Portugal

Havelock T (1955) Waves due to a floating sphere making periodic heaving oscillations. P Roy Soc A Math Phy 231(1184):1-7

Mavrakos SA, McIver P (1997) Comparison of methods for computing hydrodynamic characteristics of arrays of wave power devices. Appl Ocean Res 19(5-6):283-291 
McGuinness JPL, Thomas G (2015) Optimal arrangements of elementary arrays of wave-power devices. In: Eleventh European Wave and Tidal Energy Conference. Nantes, France

McIver P (1994) Some hydrodynamic aspects of arrays of wave-energy devices. Appl Ocean Res 16(2):61-69
Thomas GP, Evans DV (1981) Arrays of three-dimensional waveenergy absorbers. J Fluid Mech 108:67-88 